\title{
Three-nucleon forces and spectroscopy of neutron-rich calcium isotopes
}

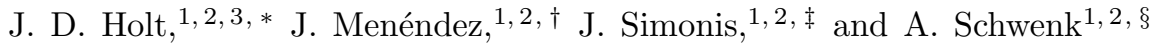 \\ ${ }^{1}$ Institut für Kernphysik, Technische Universität Darmstadt, 64289 Darmstadt, Germany \\ ${ }^{2}$ ExtreMe Matter Institute EMMI, GSI Helmholtzzentrum für Schwerionenforschung GmbH, 64291 Darmstadt, Germany \\ ${ }^{3}$ National Superconducting Cyclotron Laboratory and Department of Physics and Astronomy, \\ Michigan State University, East Lansing, MI 48844, USA
}

\begin{abstract}
We study excited-state properties of neutron-rich calcium isotopes based on chiral two- and threenucleon interactions. We first discuss the details of our many-body framework, investigate convergence properties, and for two-nucleon interactions benchmark against coupled-cluster calculations. We then focus on the spectroscopy of ${ }^{47-56} \mathrm{Ca}$, finding that with both $3 \mathrm{~N}$ forces and an extended $p f g_{9 / 2}$ valence space, we obtain a good level of agreement with experiment. We also study electromagnetic transitions and find that experimental data are well described by our calculations. In addition, we provide predictions for unexplored properties of neutron-rich calcium isotopes.
\end{abstract}

PACS numbers: 21.10.-k, 21.30.-x, 21.60.Cs, 27.40.+z

\section{INTRODUCTION}

Understanding the evolution of shell structure from the valley of stability to neutron-rich extremes represents a key challenge in nuclear structure 1, 2]. With a closed proton shell, the calcium isotopes provide an ideal region to investigate shell formation and evolution in mediummass nuclei from nuclear forces. The rich shell structure beyond ${ }^{48} \mathrm{Ca}$, combined with the capabilities of rare isotope beam facilities, has lead to an intensive focus on calcium isotopes, where new measurements provide exciting tests and constraints for state-of-the-art many-body methods and nuclear forces.

Recent experiments have established new shell closures in exotic calcium isotopes. A possible $N=32$ closure in ${ }^{52} \mathrm{Ca}$ was first recognized from a higher first-excited $2^{+}\left(2_{1}^{+}\right)$energy [3, 4], more than $1.5 \mathrm{MeV}$ higher than the $2_{1}^{+}$energy in the neighboring ${ }^{50} \mathrm{Ca}$. Moreover, signatures of a $N=32$ magic number were discovered in nearby titanium and chromium isotopes [5] 8 . These observations have been complemented by high-precision mass measurements, which revealed a flat behavior of the two-neutron separation energy, $S_{2 n}$, leading up to the shell closure at ${ }^{52} \mathrm{Ca}$ [9]. Groundbreaking mass measurements out to ${ }^{54} \mathrm{Ca} 10$ discovered a steep decrease in $S_{2 n}$ from ${ }^{52} \mathrm{Ca}$ to ${ }^{54} \mathrm{Ca}$. Combined with the high two-neutron shell-gap $S_{2 n}(Z, N)-S_{2 n}(Z, N+2)$, this unambiguously established $N=32$ as a prominent shell closure [10]. Evidence for a $N=34$ shell closure has proven more elusive, as the $2_{1}^{+}$energy is not high in the neighboring titanium or chromium isotopes [11-14]. Very recently, the $2_{1}^{+}$energy in ${ }^{54} \mathrm{Ca}$ was found to be only $\sim 500 \mathrm{keV}$ below that in ${ }^{52} \mathrm{Ca}[15]$, suggesting a new shell closure. Mass measurements through ${ }^{56} \mathrm{Ca}$ will be essential to establish the closed-shell nature of ${ }^{54} \mathrm{Ca}$. In addition, the

\footnotetext{
* jason.holt@physik.tu-darmstadt.de

$\dagger$ javier.menendez@physik.tu-darmstadt.de

$\ddagger$ simonis@theorie.ikp.physik.tu-darmstadt.de

$\S$ schwenk@physik.tu-darmstadt.de
}

spectroscopy of neutron-rich calcium isotopes provides valuable information, with important tests of theoretical calculations 16 18.

Previous work for the calcium isotopes includes phenomenological valence-space [19 23] or beyond-meanfield calculations 24. While these approaches are generally successful in reproducing experiment up to ${ }^{52} \mathrm{Ca}$, disagreement is significant in ${ }^{53,54} \mathrm{Ca}$, which led to many experimental and theoretical efforts aiming to clarify the nature of $N=34$ in calcium. The uncertainty in extrapolating phenomenological models to exotic nuclei shows the importance of developing systematic many-body approaches based on nuclear forces. Such calculations were initially pursued based on two-nucleon (NN) forces, but failed to reproduce the standard $N=28$ shell closure and other key features in calcium for $A \gtrsim 47$ [19, 25]. Neglected three-nucleon $(3 \mathrm{~N})$ forces were suggested to be the crucial missing ingredient [26].

The calcium region currently represents a frontier for ab initio calculations based on $\mathrm{NN}$ and $3 \mathrm{~N}$ forces [27]. The first application of $3 \mathrm{~N}$ forces in calcium was in the context of valence-space Hamiltonians [28, which demonstrated the important role of $3 \mathrm{~N}$ forces in reproducing the dripline and spectra in oxygen isotopes 22, 30, as well as in proton-rich nuclei [31. In calcium isotopes, $3 \mathrm{~N}$ forces provided the first microscopic explanation for the $N=28$ magic number in ${ }^{48} \mathrm{Ca}$, as well as for the ground-state energies [28]. The improved calculations of Refs. [9, 10, 32] successfully predicted the additional binding found in ${ }^{52} \mathrm{Ca}$ [9] as well as the behavior of the two-neutron separation energy from ${ }^{48-54} \mathrm{Ca}$ [10]. Coupled-cluster (CC) calculations, including continuum degrees of freedom and phenomenological $3 \mathrm{~N}$ forces, also found a very good description of these signatures 33 . This agreement extends to ab initio self-consistent Green's function (SCGF) calculations with chiral $\mathrm{NN}$ and $3 \mathrm{~N}$ forces [34, 35]. In addition, the $\mathrm{CC}$ calculations of Ref. 36] and the ab initio in-medium similarity renormalization group (IMSRG) [37, 38, have been applied to the calcium isotopes.

In this paper, we present a comprehensive study of excited-state properties of neutron-rich calcium isotopes 
based on our valence-space approach of Refs. [9, 10, 28, 32 , focusing in particular on shell structure in the region around $N=28,32,34$. In Sect. II], we discuss details of the calculation of valence-space Hamiltonians perturbatively, based on $\mathrm{NN}$ and $3 \mathrm{~N}$ forces from chiral effective field theory (EFT) 39, 40]. We investigate convergence both order-by-order in many-body perturbation theory and in terms of intermediate-state excitations, with NN interactions evolved to low momentum via renormalization group (RG) methods. For NN interactions, we benchmark against $\mathrm{CC}$ calculations and find reasonable agreement. In Sect. III, we calculate spectra and electromagnetic transitions in ${ }^{47-56} \mathrm{Ca}$, showing that with $3 \mathrm{~N}$ forces and an extended valence space, good agreement with experiment is obtained, in many cases comparable to phenomenological interactions. Finally, we explore the role of residual $3 \mathrm{~N}$ forces. Similar to the oxygen isotopes [4], their impact on spectra is minor, while for ground-state energies their contributions increase with the number of valence nucleons.

\section{MICROSCOPIC VALENCE-SPACE HAMILTONIANS}

\section{A. Many-body perturbation theory}

For a given nucleus, the solution of the $A$-body Schrödinger equation with the Hamiltonian $H$ gives the eigenstates $\left|\psi_{n}\right\rangle$ and energies $E_{n}$,

$$
H\left|\psi_{n}\right\rangle=\left(H_{0}+V\right)\left|\psi_{n}\right\rangle=E_{n}\left|\psi_{n}\right\rangle,
$$

where $H_{0}$ defines a single-particle basis $\left|\phi_{n}\right\rangle$ with corresponding eigenvalues $\epsilon_{n}$,

$$
H_{0}\left|\phi_{n}\right\rangle=\epsilon_{n}\left|\phi_{n}\right\rangle
$$

and $V$ includes the interactions between nucleons. Solving the $A$-body Schrödinger equation in a large singleparticle basis by diagonalization is challenging due to the large number of configurations involved. Therefore, many-body methods generally take one of two strategies to describe medium-mass nuclei. In approaches such as CC theory [33, 36, 42, SCGF theory [34, 35], or the IMSRG [37, 38, all nucleons are active, but some truncations are necessary in practice. In valence-space methods, the number of degrees of freedom is reduced by treating the nucleus as a many-body system comprised of a closed-shell core, with the additional nucleons occupying a truncated single-particle (valence) space. After deriving an effective valence-space Hamiltonian, this is then diagonalized exactly in the valence space.

We first define operators $P$ and $Q$, which project into and out of the valence space, respectively,

$$
\begin{aligned}
& P=\sum_{i=1}^{d}\left|\phi_{i}\right\rangle\left\langle\phi_{i}\right|, \\
& Q=1-P,
\end{aligned}
$$

where $d$ is the dimension of the valence space, $P^{2}=P$, $Q^{2}=Q$, and $P Q=0$. Then, the goal is to construct an effective valence-space Hamiltonian, $H_{\text {eff }}$,

$$
P H_{\mathrm{eff}} P\left|\psi_{\alpha}\right\rangle=E_{\alpha} P\left|\psi_{\alpha}\right\rangle
$$

with

$$
H_{\mathrm{eff}}=\sum_{i=1}^{d} \varepsilon_{i} a^{\dagger} a+V_{\mathrm{eff}}
$$

which after diagonalization in the valence space reproduces a subset $E_{\alpha}$ of eigenvalues of the full $A$-body Hamiltonian. Here, $\varepsilon_{i}$ are the single-particle energies (SPEs) of the $d$ orbitals in the valence space and $V_{\text {eff }}$ is the effective interaction between valence nucleons.

Many-body perturbation theory (MBPT) provides a diagrammatic framework to calculate both the SPEs $\varepsilon_{i}$ and $V_{\text {eff }}$ from nuclear forces [25, 43,45]. This approach has been pursued with NN interactions, but due to poor agreement with experiment, all shell model calculations in practice involve adjustments of either the SPEs, $V_{\text {eff }}$, or both. To calculate $H_{\text {eff }}$ we start from an energydependent effective interaction between valence nucleons, the $\widehat{Q}$-box, which takes into account excitations outside the valence space,

$$
\widehat{Q}(\omega)=P V P+P V Q \frac{1}{\omega-Q H Q} Q V P,
$$

and is evaluated at the unperturbed starting energy $\omega=P H_{0} P$. The diagrammatic expansion of $\widehat{Q}$ consists of all irreducible, valence-linked diagrams. To remove the energy dependence, we include folded diagrams through a nonperturbative transformation involving $\widehat{Q}$ and its energy derivatives. This results in an energy-independent, size-extensive effective interaction

$$
V_{\mathrm{eff}}^{(k)}=\widehat{Q}+\sum_{m=1}^{\infty} \frac{1}{m !}\left(\frac{\mathrm{d}^{m} \widehat{Q}}{\mathrm{~d} \omega^{m}}\right)\left(V_{\mathrm{eff}}^{(k-1)}\right)^{m} .
$$

This integral equation is solved by iteration, which converges when $V_{\mathrm{eff}}^{(k)} \approx V_{\mathrm{eff}}^{(k-1)}$, typically after $\sim 15$ iterations. We make two approximations in our evaluation of $\widehat{Q}$. First we truncate $\widehat{Q}$ at some finite order. In this work we include contributions up to third order in MBPT, the current state-of-the art. Second, excitations out of the valence space are allowed to some finite energy $N \hbar \omega$, which is ultimately limited by the size of the single-particle basis. Convergence with respect to these two approximations is discussed in Sect. II C.

We then calculate the SPEs in ${ }^{41} \mathrm{Ca}$ consistently from the corresponding set of one-body diagrams, the $\widehat{S}$-box, also taken to third order in MBPT in the same harmonicoscillator basis as the $\widehat{Q}$-box. To obtain the singleparticle energies, we solve the coupled Dyson equations,

$$
\varepsilon_{i}^{(k)}=\left\langle i\left|H_{0}\right| i\right\rangle+\widehat{S}_{i}\left(\varepsilon_{i}^{(k-1)}\right),
$$



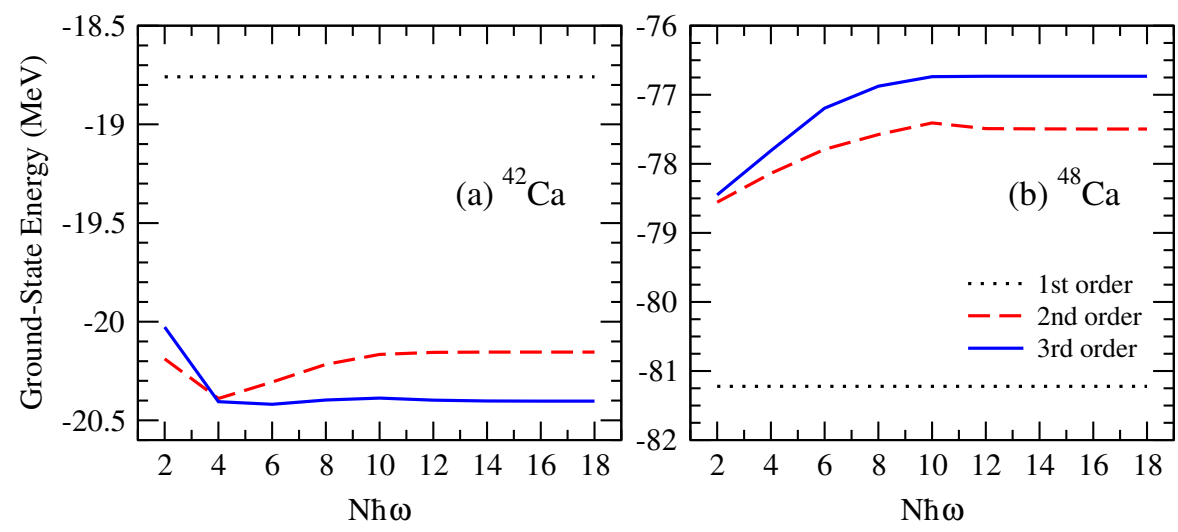

FIG. 1. (Color online) Convergence of the (a) ${ }^{42} \mathrm{Ca}$ and (b) ${ }^{48} \mathrm{Ca}$ ground-state energies as a function of increasing intermediatestate excitations $N \hbar \omega$ and perturbative order. Calculations are based on NN forces in 13 major harmonic-oscillator shells.

by iteration starting from $\varepsilon_{i}^{(0)}=\omega$ until $\varepsilon_{i}^{(k)} \approx \varepsilon_{i}^{(k-1)}$. Because the $\varepsilon_{i}$ depend on relative shifts in the unperturbed harmonic-oscillator spectrum, we also update the unperturbed valence-space energy to be the centroid of the converged SPEs and iterate until the centroid of the final SPEs is equal to the unperturbed value. Convergence is reached after $\sim 10$ iterations.

\section{B. Nuclear interactions}

Our results are based on nuclear forces derived in chiral EFT, a systematic expansion for nuclear forces [39, 40] in which $3 \mathrm{~N}$ interactions arise naturally at next-to-nextto-leading order $\left(\mathrm{N}^{2} \mathrm{LO}\right)$. At the $\mathrm{NN}$ level, we perform an RG evolution [46, 47] of the $500 \mathrm{MeV} \mathrm{N}{ }^{3} \mathrm{LO}$ NN potential of Ref. 48. using a smooth regulator [49] with $\Lambda=2.0 \mathrm{fm}^{-1}$, to obtain a low momentum interaction, $V_{\text {low } k}$. The RG evolution decouples low from high momenta and improves the convergence of the MBPT calculation [47. At the $3 \mathrm{~N}$ level, we use the leading $\mathrm{N}^{2} \mathrm{LO}$ $3 \mathrm{~N}$ forces 50, 51, which include a long-range two-pionexchange part, a shorter-range one-pion exchange, and a $3 \mathrm{~N}$ contact interaction. The two undetermined $3 \mathrm{~N}$ couplings are fit to the ${ }^{3} \mathrm{H}$ binding energy and the ${ }^{4} \mathrm{He}$ radius at the same resolution scale as the $V_{\text {low } k}$ interaction [52].

In the calculation of valence-space Hamiltonians, we include normal-ordered one- and two-body parts of $3 \mathrm{~N}$ forces, which correspond to interactions among one valence and two core nucleons, or two valence and one core nucleon, respectively. These give rise to repulsive interactions among valence neutrons and increase the spinorbit splitting in the SPEs 28, 29. They are expected to be dominant over residual $3 \mathrm{~N}$ forces between three valence particles because of phase-space considerations [53]. This has been confirmed in CC calculations of light and medium-mass nuclei [36, 54. In Sect. IIID, we explore this by calculating the contributions from residual $3 \mathrm{~N}$ forces [10, 41] for ground and first-excited states.

We work in a harmonic-oscillator basis with $\hbar \omega=$

\begin{tabular}{cccc}
\hline \hline Orbital & \multicolumn{2}{c}{ Phenomenological } & MBPT \\
& GXPF1A [22] & KB3G [20] & $\mathrm{pfg}_{9 / 2}$ \\
\hline $\mathrm{f}_{7 / 2}$ & -8.62 & -8.60 & -8.05 \\
$\mathrm{p}_{3 / 2}$ & -5.68 & -6.60 & -5.86 \\
$\mathrm{p}_{1 / 2}$ & -4.14 & -4.60 & -3.22 \\
$\mathrm{f}_{5 / 2}$ & -1.38 & -2.10 & -1.33 \\
$\mathrm{~g}_{9 / 2}$ & $(-1.00)$ & - & -1.23 \\
\hline \hline
\end{tabular}

TABLE I. Phenomenological and calculated (MBPT) SPEs in $\mathrm{MeV}$. Details are given in the text.

$11.48 \mathrm{MeV}$ and include $\mathrm{NN}$ forces in 13 major shells and $3 \mathrm{~N}$ forces in 5 major shells. When $3 \mathrm{~N}$ forces are included fully to third order in MBPT, we find that the contribution to SPEs range from $\sim 1-6 \mathrm{MeV}$ for different orbitals, approximately an order of magnitude larger than the effects on valence-space interactions, as expected from the hierarchy of normal-ordered contributions 54. Moreover, with $3 \mathrm{~N}$ forces, the calculated SPEs are comparable to the empirical values of the phenomenological GXPF1A [22] and KB3G 20] interactions for the $p f$-shell orbitals [28, 32], as shown in Table I]

\section{Convergence properties}

Next, we discuss the convergence properties of MBPT when using low-momentum NN interactions. For a fixed valence space, the results must converge order-by-order in the $\widehat{Q}$ - and $\widehat{S}$-box expansion as well as in the allowed intermediate-state excitations. Because the RG evolution renders nuclear interactions more perturbative and decouples low- from high-oscillator states, improved convergence behavior is expected in both respects [47.

Assessing order-by-order convergence beyond third order is a challenge for MBPT, since complete fourth-order calculations are beyond current computational capabilities and have not been attempted. In Fig. 1, we show the order-by-order convergence of the ${ }^{42,48} \mathrm{Ca}$ ground- 

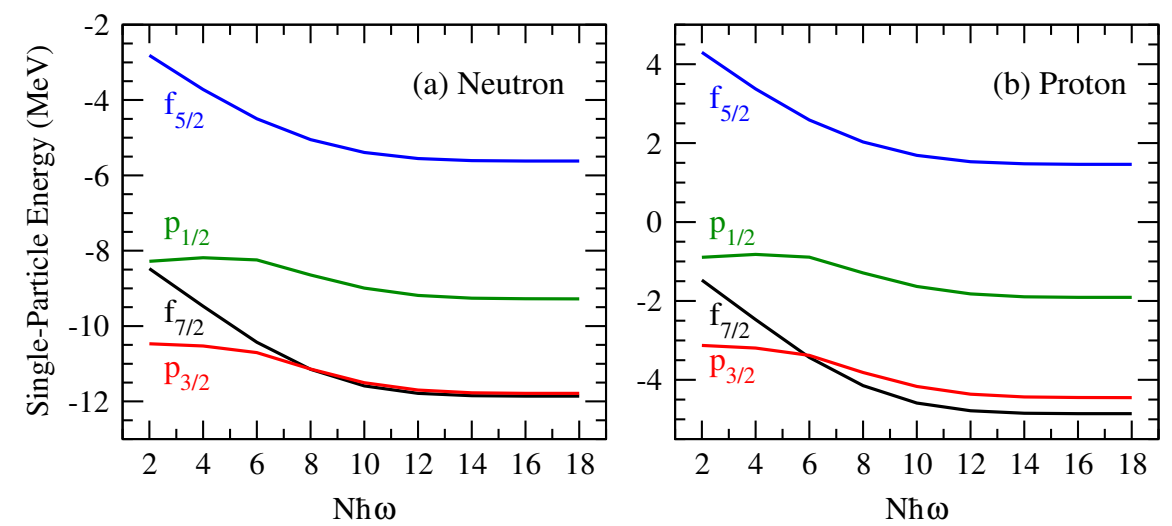

FIG. 2. (Color online) Convergence of (a) neutron and (b) proton SPEs as a function of increasing intermediate-state excitation $N \hbar \omega$. Calculations are based on NN forces in 13 major harmonic-oscillator shells.

state energies of ${ }^{42,48} \mathrm{Ca}$ as a function of increasing intermediate-state excitations $N \hbar \omega$, within a harmonicoscillator basis of 13 major shells. N$N \omega$ denotes the number of excitation quanta in a given intermediatestate configuration (e.g., two neutrons excited three shells above the valence space is a $6 \hbar \omega$ excitation). In these studies, we use NN-only forces with empirical SPEs and see promising order-by-order behavior: the change from second to third order is $\sim 15 \%$ of the change from first to second order. While the calculations cannot be said to be completely converged at third order, this trend suggests that changes due to a complete fourth-order calculation would be small. In particular, they will be less important than other uncertainties in the calculation, such as the uncertainties in the input Hamiltonian.

We also observe in Fig. 1 that in terms of intermediatestate excitations, the convergence with $V_{\text {low } k}$ is rapid, with 3rd order converging faster than 2 nd order. For all orders, the ground-state energies of both ${ }^{42,48} \mathrm{Ca}$ are well converged by $\sim 12 \hbar \omega$. Similarly, Fig. 2 shows the convergence of neutron and proton SPEs in the $p f$-shell as a function of $N \hbar \omega$. While convergence is slower compared to the ground-state energies, all SPEs are converged by $14 \hbar \omega$, with neutron and proton SPEs following a very similar convergence pattern. Finally, all calculations with $3 \mathrm{~N}$ forces seem to be converged when included in 5 major shells. In two-body matrix elements and SPEs, the change from four to five major shells is $\sim 10 \mathrm{keV}$ and $\sim 50 \mathrm{keV}$, respectively. Work to extend $3 \mathrm{~N}$ forces beyond 5 major shells is currently in progress.

\section{Benchmark with coupled-cluster theory}

We can also benchmark the MBPT energies with ab initio methods using identical starting interactions and working in the same single-particle basis. Here, we perform CC calculations for the ground-state energies of the calcium isotopes using the same $V_{\text {low } k}$ interaction in a single-particle basis of 13 major harmonic-oscillator

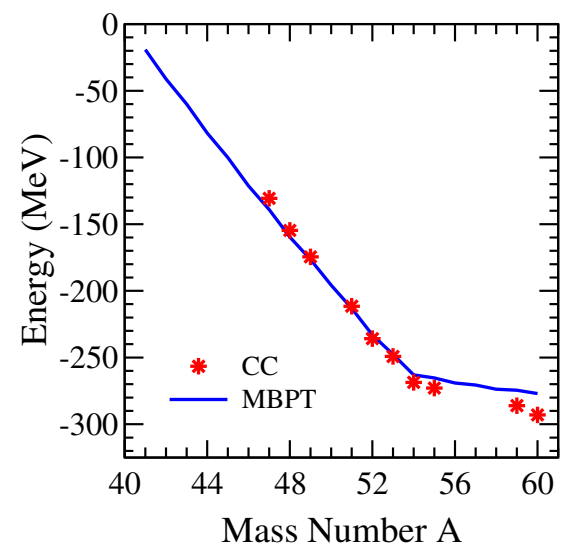

FIG. 3. (Color online) Comparison of MBPT and CC groundstate energies of calcium isotopes relative to ${ }^{40} \mathrm{Ca}$ based on the same NN interaction (for details see text). The MBPT results use the SPEs obtained in CC theory.

shells with $\hbar \omega=12 \mathrm{MeV}$. The results are shown in Fig. 3 relative to the ground-state energy of ${ }^{40} \mathrm{Ca}$. The closed $j$-subshell systems, ${ }^{40,48,52,54,60} \mathrm{Ca}$, are calculated at the $\Lambda$-CCSD $(\mathrm{T})$ level [42, 55]. The $A \pm 1$ systems, $47,49,51,53,55,59 \mathrm{Ca}$, are obtained with the CC particleattached/removed equations of motion method at the singles and doubles level (PA/PR-EOM-CCSD) 42, 55.

To compare with CC results, we perform the MBPT calculations in the $p f$ shell, where the SPEs are taken as the PA-EOM-CCSD $\left(f_{7 / 2}, p_{3 / 2}, p_{1 / 2}, f_{5 / 2}\right)$ energies in ${ }^{41} \mathrm{Ca}$. The particle-attached $g_{9 / 2}$ is not of single-particle character, so the MBPT $p f$-shell comparison provides the cleanest benchmark. This comparison probes the twobody part of the valence-space Hamiltonian, assessing the reliability of the convergence trend illustrated in Fig. 1.

In Fig. 3, we find that the MBPT ground-state energies are within $5 \%$ (in most cases much better) of those of CC theory. This shows that MBPT can be comparable to CC theory for $V_{\text {low } k}$ interactions, provided that consistent SPEs are employed. 
While the CC ground-state energies agree well with MBPT to ${ }^{55} \mathrm{Ca}$, this agreement deteriorates for heavier isotopes. The reason is that the $\mathrm{CC}$ calculations begin to fill the $g_{9 / 2}$ orbit, which is lower in energy than the calculated $f_{5 / 2}$. This makes a comparison of the CC and $p f$-shell valence-space calculations unreliable for ${ }^{59,60} \mathrm{Ca}$. Moreover, a benchmark in the $p f g_{9 / 2}$ space is not possible, because, as mentioned, the CC one-particle attached $g_{9 / 2}$ state in ${ }^{41} \mathrm{Ca}$ is not of single-particle character.

\section{E. Valence-space calculations}

For neutron-rich oxygen and calcium isotopes, we have shown that it is necessary in MBPT calculations of valence-space Hamiltonians to expand the valence space beyond the standard one major harmonic-oscillator shell [28, 30 32]. This takes into account the effects of the additional orbitals nonperturbatively, so that the general strategy is to make the valence space for diagonalization as large as possible and include the contributions beyond the valence space in MBPT, which converges better for larger valence spaces.

In this work, we perform calculations in both the $0 f_{7 / 2}$, $1 p_{3 / 2}, 0 f_{5 / 2}, 1 p_{1 / 2}$ valence space ( $p f$ shell) and the extended space including the $0 g_{9 / 2}$ orbit $\left(p f g_{9 / 2}\right.$ valence space), in both cases on top of a ${ }^{40} \mathrm{Ca}$ core. We take two approaches with respect to SPEs: in all $p f$-shell calculations we use the empirical GXPF1A SPEs, while for the $p f g_{9 / 2}$ space we either use the GXPF1A values (setting $g_{9 / 2}=-1.0 \mathrm{MeV}$ ), or the MBPT SPEs calculated consistently, as shown in Table I The shell model codes ANTOINE [19, 56] and NATHAN [19] have been used throughout this work.

The $p f g_{9 / 2}$ space consists of orbitals beyond one major harmonic-oscillator shell, which means that the centerof-mass $(\mathrm{cm})$ motion of the valence nucleons will not factorize in general. Following Refs. [57, 58, we have investigated possible center-of-mass $(\mathrm{cm})$ contamination in our calculations by adding a cm Hamiltonian, $\beta H_{c m}$, with $\beta=0.5$, to our original Hamiltonian. This has a modest impact on excitation spectra, where states can be affected up to $\sim 200 \mathrm{keV}$. This difference can be understood because the non-zero cm two-body matrix elements are also relevant matrix elements of the MBPT calculation, and a clear separation between these two effects is difficult. Similarly, we find non-negligible $\left\langle H_{c m}\right\rangle$ values, which point to possible $\mathrm{cm}$ admixture and/or nonnegligible occupancies of the $g_{9 / 2}$ orbital.

There are several directions in progress to investigate this further in both the $p f g_{9 / 2}$ and $s d f_{7 / 2} p_{3 / 2}$ [30] spaces. We will carry out a nonperturbative Okubo-Lee-SuzukiOkamoto transformation 60, 61] into the standard onemajor-shell space, which is free of $\mathrm{cm}$ spurious states. This will keep the treatment of the orbitals within the extended space nonperturbative, while treating the MBPT configurations perturbatively. We will also apply the IMSRG [37] to extended valence spaces, tailoring the evo- lution so that the cross-shell matrix elements have small values, $\left\langle H_{c m}\right\rangle \rightarrow 0$. Finally, we will explore different valence spaces, choosing the core of the calculations so that the $\mathrm{cm}$ factorizes. For instance, for the neutronrich calcium isotopes a ${ }^{48} \mathrm{Ca}$ core can be used. Here, we follow the calculations of ground-state energies of Refs. [9, 10, 32] and present results for the spectra for the same interactions.

\section{RESULTS}

\section{A. Ground-state energies}

The calculated ground-state energies for calcium isotopes are shown for both the $p f$ and $p f g_{9 / 2}$ shells in Fig. 4. These are the same as for the predictions of the neutron-rich ${ }^{51-54} \mathrm{Ca}$ reported in Ref. [9, 10]. They update the results of Ref. [28, where $3 \mathrm{~N}$ forces where included only to first order in MBPT. The repulsive effect of normal-ordered $3 \mathrm{~N}$ forces [28, 29] is evident in both valence spaces, and there is only a small difference between the calculations with empirical and calculated (MBPT) SPEs, which reflects the similar values shown in Table [.

While the $p f$ and $p f g_{9 / 2}$ spaces give similar absolute ground-state energies, detailed comparisons to recent experimental two-neutron separation energies [9, 10] and three-point mass differences [9, 32 highlight the good agreement found with the $p f g_{9 / 2}$-shell results. Beyond ${ }^{60} \mathrm{Ca}$ the ground-state energies evolve very flat with $A$, which makes a precise prediction of the dripline difficult. Moreover, for masses beyond ${ }^{54} \mathrm{Ca}, \mathrm{CC}$ calculations indicate that continuum degrees of freedom play an important role in lowering the $1 d_{5 / 2}$ and $2 s_{1 / 2}$ orbitals, not included in our calculations. As a result these orbitals may become degenerate with $0 g_{9 / 2}$ near ${ }^{60} \mathrm{Ca}$, and further lowering of the ground-state energies beyond ${ }^{60} \mathrm{Ca}$ is expected 33. Therefore, to explore reliably the neutronrich region towards the dripline, continuum degrees of freedom and larger valence spaces are necessary.

\section{B. Spectra}

We now calculate the spectra of neutron-rich calcium isotopes, comparing our MBPT predictions to experiment when available, as well as to shell model results using the phenomenological interactions GXPF1A 22 and $\mathrm{KB} 3 \mathrm{G}$ [20. We discuss in detail the spectra of the neutron-rich isotopes ${ }^{47-56} \mathrm{Ca}$. For ${ }^{47-50} \mathrm{Ca}$ we present different calculations based on chiral interactions and compare to experiment, emphasizing the importance of extended valence spaces and $3 \mathrm{~N}$ forces. To quantify these effects in excitation spectra, we show results with $\mathrm{NN}$ forces only and $\mathrm{NN}+3 \mathrm{~N}$ forces, in the $p f$-shell and in the extended $p f g_{9 / 2}$ space. For ${ }^{51-56} \mathrm{Ca}$, where there is none or limited experimental information on excited states, we focus on the predictions of our best calculations $(\mathrm{NN}+3 \mathrm{~N}$ 

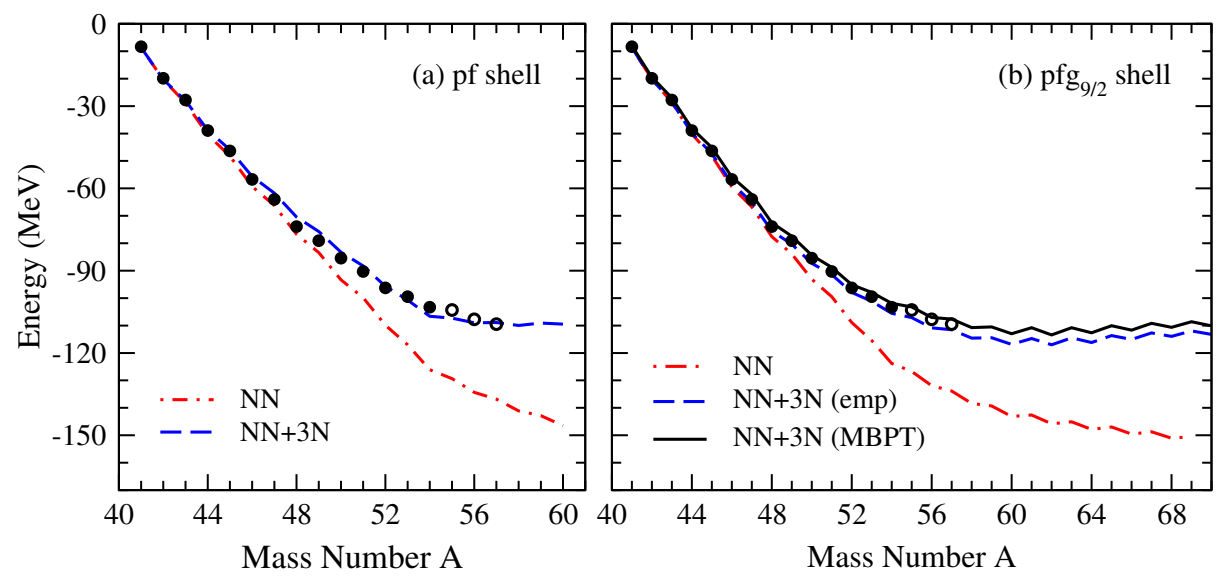

FIG. 4. (Color online) Calculated ground-state energies of calcium isotopes in (a) $p f$ shell and (b) $p f g_{9 / 2}$ shell, compared with experimental data (solid points) and AME2012 extrapolated values (open circles) [59. Calculations are performed in the extended $p f g_{9 / 2}$ valence space and based on $\mathrm{NN}$ forces only, $\mathrm{NN}+3 \mathrm{~N}$ forces with empirical $\mathrm{SPEs}$, and $\mathrm{NN}+3 \mathrm{~N}$ forces with calculated (MBPT) SPEs.

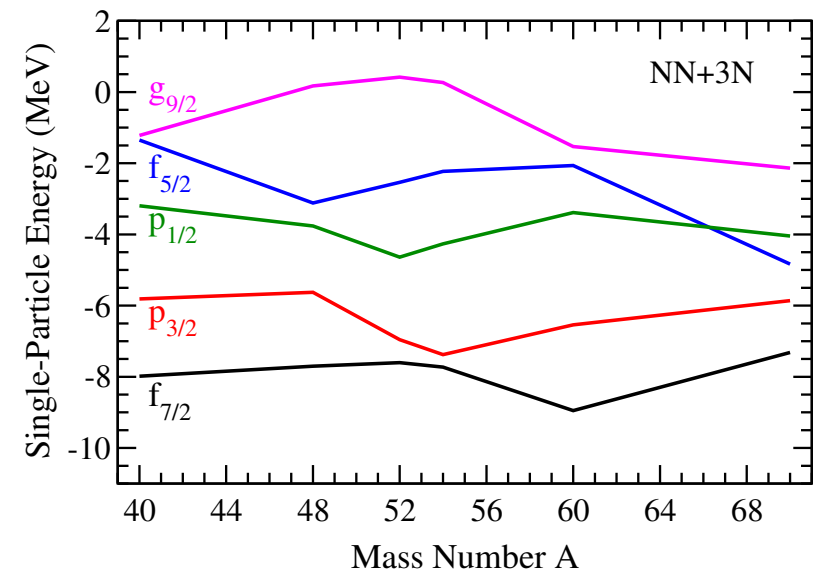

FIG. 5. (Color online) Evolution of SPEs as a function of mass number. Calculations are based on $\mathrm{NN}+3 \mathrm{~N}$ forces in the extended $p f g_{9 / 2}$ space.

forces in the $p f g_{9 / 2}$ space). The spectra for the lighter ${ }^{42-46} \mathrm{Ca}$, which mostly probe the $f_{7 / 2}$ orbital, are given in Appendix A

To understand these results, we refer to the effective single-particle energies (ESPEs), given in Fig. 5, for $\mathrm{NN}+3 \mathrm{~N}$ forces in the $p f g_{9 / 2}$ space, which describe the evolution of the spherical mean field of the calculation. While correlations are important in the final results and are included via exact diagonalization, ESPEs provide a guide to the position of different orbitals for a given neutron number within our valence-space framework. The important role of the extended valence spaces is manifested in the occupancies of the $g_{9 / 2}$ orbital. For groundstates, these become significant in ${ }^{47} \mathrm{Ca}$, with $g_{9 / 2}$ occupation number 1.2 , increasing to 2.1 in ${ }^{56} \mathrm{Ca}$. They are accompanied by a depletion of the $f_{7 / 2}$ occupations.

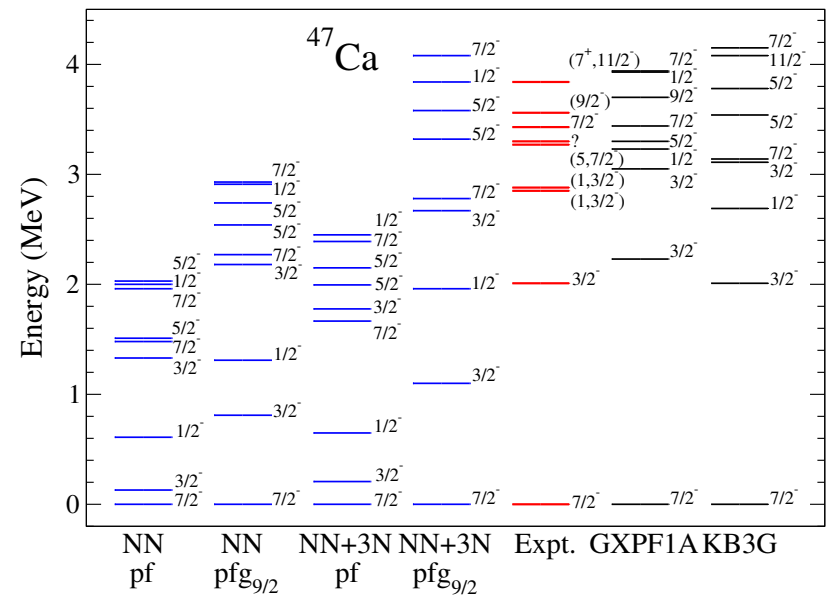

FIG. 6. (Color online) Excitation energies of bound excited states in ${ }^{47} \mathrm{Ca}$ compared with experiment 62 and phenomenological GXPF1A 22] and KB3G 20] interactions. The NN-only results are calculated in the $p f$ and $p f g_{9 / 2}$ spaces with empirical SPEs. The $\mathrm{NN}+3 \mathrm{~N}$ results are obtained in the same spaces. In the $p f$-shell, empirical SPEs are used, while the $p f g_{9 / 2}$ space results use the consistently calculated MBPT SPEs.

$$
\text { 1. }{ }^{47} \mathrm{Ca}
$$

In Fig. 6 we show the calculated spectra for ${ }^{47} \mathrm{Ca}$ in the $p f$ and $p f g_{9 / 2}$ spaces using NN-only and $\mathrm{NN}+3 \mathrm{~N}$ forces. In the $p f$-shell calculations, the spectra are too compressed. The two lowest-lying states differ by only $200 \mathrm{keV}$, and there is otherwise very poor agreement with experiment. Furthermore, the effects of $3 \mathrm{~N}$ forces in the $p f$-shell are relatively small. Extending the calculation to the $p f g_{9 / 2}$ space with $\mathrm{NN}$ forces only partially improves the spectrum, but it remains too compressed. Our final 


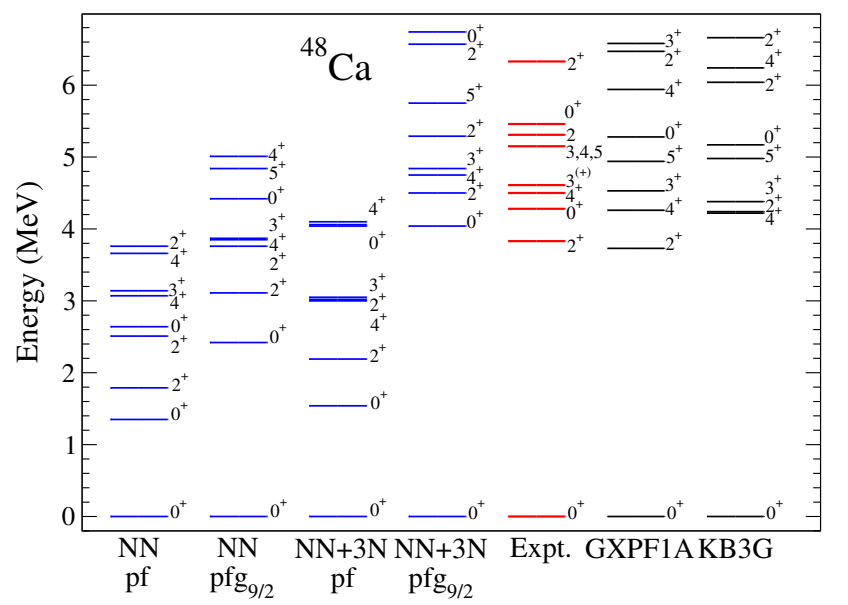

FIG. 7. (Color online) Excitation energies of bound excited states in ${ }^{48} \mathrm{Ca}$ compared with experiment 62 and phenomenological interactions (labels as in Fig. 6.

results with $\mathrm{NN}+3 \mathrm{~N}$ forces in the extended space improve the spectrum, leading to the best agreement with experiment.

Nevertheless, we still observe deficiencies in our MBPT spectrum. The major disagreement is in the lowest $3 / 2^{-}$ state, which is approximately $1 \mathrm{MeV}$ below experiment, reflecting the small $f_{7 / 2}-p_{3 / 2}$ gap around ${ }^{48} \mathrm{Ca}$, as seen in Fig. 5. This state is well reproduced by the phenomenological interactions. Similarly, the $1 / 2_{1}^{-}$state is also low, due to the small $f_{7 / 2}-p_{1 / 2}$ gap in our calculations.

\section{2. ${ }^{48} \mathrm{Ca}$}

Figure 7 shows the calculated ${ }^{48} \mathrm{Ca}$ spectra compared with experiment. As with ${ }^{47} \mathrm{Ca}$ we note that the $p f$ space generally gives too compressed spectra, and $3 \mathrm{~N}$ forces give only minor improvements. In the extended $p f g_{9 / 2}$ space, while $\mathrm{NN}$ forces also give a poor experimental description, significant improvement is obtained when $3 \mathrm{~N}$ forces are included.

The gap between the ground state and the $2_{1}^{+}$state, a measure of the shell closure at $N=28$, is well reproduced, though somewhat overpredicted by $500 \mathrm{keV}$. As we have a relatively small $f_{7 / 2}-p_{3 / 2}$ gap in the ESPEs in Fig. 5 , the high $2_{1}^{+}$state is a result of correlations involving $g_{9 / 2}$, in particular $f_{7 / 2}-g_{9 / 2}$. On the other hand, we find a $0^{+}$as the first excited state, contrary to experiment, in all calculations. Since this state is dominated by the $2 p-2 h$ configuration with respect to the ground state of the form $\left(f_{7 / 2}\right)^{-2}\left(p_{3 / 2}\right)^{2}$, this may be related to a too strong $f_{7 / 2}-p_{3 / 2}$ pairing interaction. Other excited states are in good agreement with experiment, and comparable to the results of GXPF1A and KB3G.

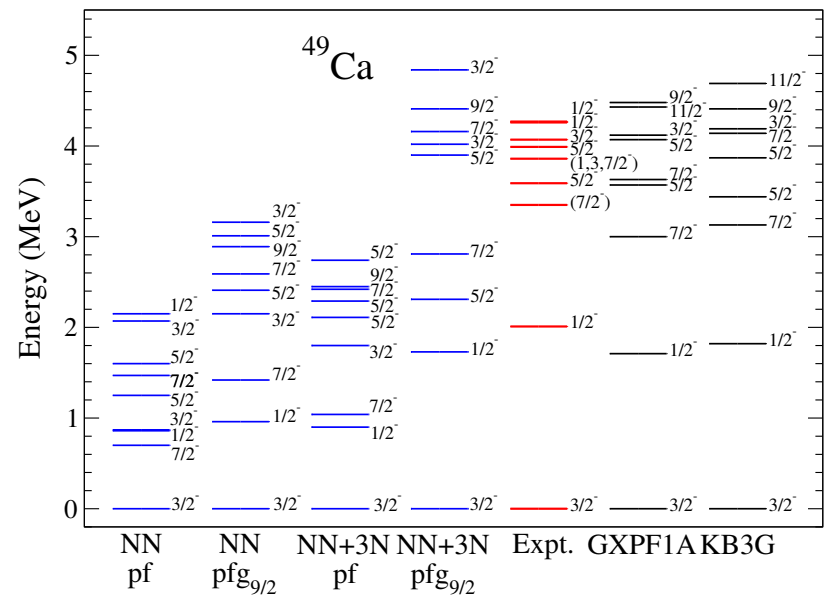

FIG. 8. (Color online) Excitation energies of bound excited states in ${ }^{49} \mathrm{Ca}$ compared with experiment [18, 62] and phenomenological interactions (labels as in Fig. 6).

$$
\text { 3. }{ }^{49} \mathrm{Ca}
$$

As in lighter isotopes, our calculations of ${ }^{49} \mathrm{Ca}$ in Fig. 8 show that with either NN forces only, or in the $p f$ shell, the physics necessary to reproduce the spectrum is not adequately captured; the excited states are too compressed and with incorrect ordering. It is only in the $\mathrm{NN}+3 \mathrm{~N}$ calculations in the extended $p f g_{9 / 2}$ space that we observe a reasonable description of the ${ }^{49} \mathrm{Ca}$ spectrum.

The ground state in ${ }^{49} \mathrm{Ca}$ is dominated by the singleparticle configuration of a $p_{3 / 2}$ particle on top of ${ }^{48} \mathrm{Ca}$. Therefore, the first excited $1 / 2_{1}^{-}$state, predicted in very good agreement with experiment, reflects the effective $p_{3 / 2}-p_{1 / 2}$ gap for this nucleus. Also the location of the lowest $7 / 2_{1}^{-}$state is in reasonable agreement with the tentatively assigned experimental level (it lies some $500 \mathrm{keV}$ lower), and with predictions from the phenomenological interactions. This state is dominated by a $2 p-1 h$ $\left(f_{7 / 2}\right)^{-1}\left(p_{3 / 2}\right)^{2}$ configuration on top of ${ }^{48} \mathrm{Ca}$, and therefore reflects the effective $f_{7 / 2}-p_{3 / 2}$ gap plus correlations discussed for the closure of ${ }^{48} \mathrm{Ca}$.

However, in our calculations we observe that the $5 / 2_{1}^{-}$ state is quite low compared to experiment and the phenomenological interactions. This is indicative of a small effective $p_{3 / 2}-f_{5 / 2}$ gap in this region. We also note that the spin of the fourth excited state has not been experimentally identified, but that our calculations, as in phenomenology, predict it as a $7 / 2^{-}$state.

$$
\text { 4. }{ }^{50} \mathrm{Ca}
$$

In Fig. 9 we see that for ${ }^{50} \mathrm{Ca}$ the location of the first excited $2_{1}^{+}$state is overpredicted in all MBPT calculations by $\sim 500 \mathrm{keV}$. The $0^{+}$ground state and the $2_{1}^{+}$ state are dominated by $\left(p_{3 / 2}\right)^{2}$ configurations. Therefore, 


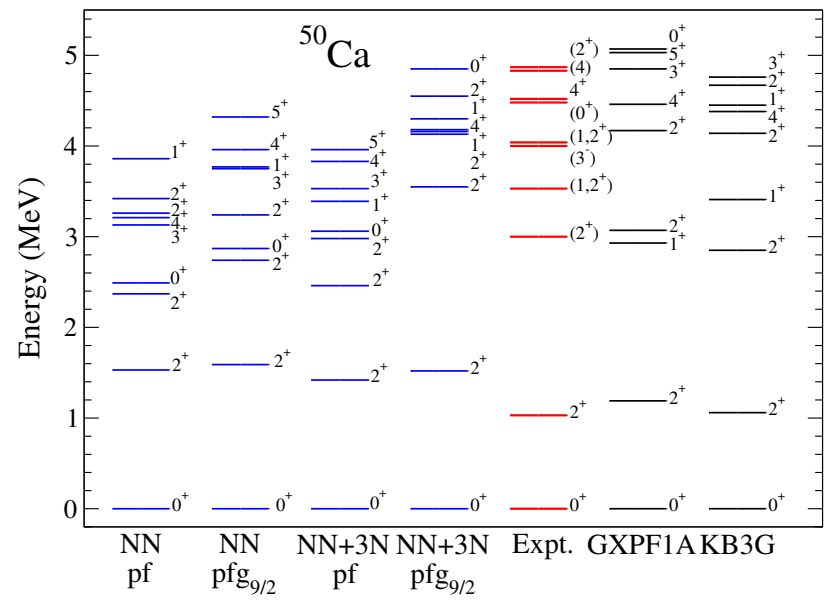

FIG. 9. (Color online) Excitation energies of bound excited states in ${ }^{50} \mathrm{Ca}$ compared with experiment [18, 62] and phenomenological interactions (labels as in Fig. 6).

the increased $2_{1}^{+}$energy is related to the low excited $0^{+}$ state found in ${ }^{48} \mathrm{Ca}$.

Although most of the experimental spin and parity assignments are tentative, in our calculations with $\mathrm{NN}+3 \mathrm{~N}$ forces in the $p f g_{9 / 2}$ space, the remaining states are compatible with experiment and comparable to the results with the phenomenological interactions. In particular the large $2 \mathrm{MeV}$ gap between the $2_{1}^{+}$and $2_{2}^{+}$states is not reproduced in our other MBPT calculations. The location of the lowest $1_{1}^{+}$state differs significantly in the three calculations, which are otherwise consistent with the data, with the MBPT prediction being $1 \mathrm{MeV}$ and $500 \mathrm{keV}$ above the GXPF1A and KB3G predictions, respectively. A reliable assignment of the spin of the third excited state in ${ }^{50} \mathrm{Ca}$ at $3.53 \mathrm{MeV}$ is needed to identify this state and test the theoretical calculations.

$$
\text { 5. }{ }^{51} \mathrm{Ca}
$$

In ${ }^{51} \mathrm{Ca}$ there is no definite experimental information on the spins of the excited states, only tentative assignments based largely on inferences from phenomenological interactions [14, 17. Therefore, we show in Fig. 10 only our $\mathrm{NN}+3 \mathrm{~N}$ calculation in the extended $p f g_{9 / 2}$ space and compare with the experimental excitation energies and with the phenomenological results.

The $3 / 2^{-}$ground state is dominated by a $p_{3 / 2}$ hole configuration below the $N=32$ subshell closure. The first excited $1 / 2^{-}$state is indicative of the effective $p_{3 / 2}-p_{1 / 2}$ gap (and approximate strength of the $N=32$ closure) and is in very good agreement with the experimental tentative spin assignment and the results of the phenomenological interactions. The $5 / 2^{-}$state with dominant $1 p-1 h\left(p_{3 / 2}\right)^{-1}\left(f_{5 / 2}\right)^{1}$ configuration above the ground state is in our calculation the $5 / 2_{1}^{-}$state, while in the phenomenological interactions it is the $5 / 2_{2}^{-}$state,

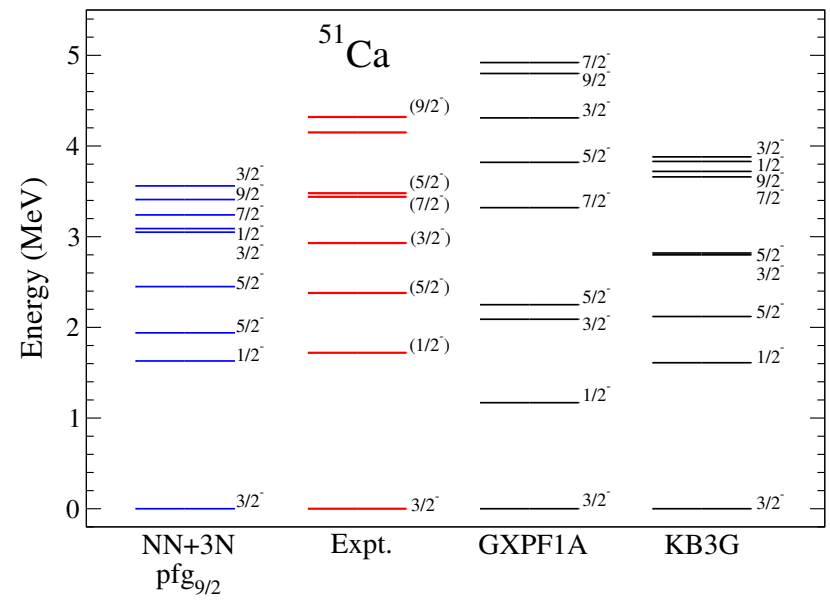

FIG. 10. (Color online) Excitation energies of bound excited states in ${ }^{51} \mathrm{Ca}$ compared with experiment [14, 17] and phenomenological interactions (labels as in Fig. 67.

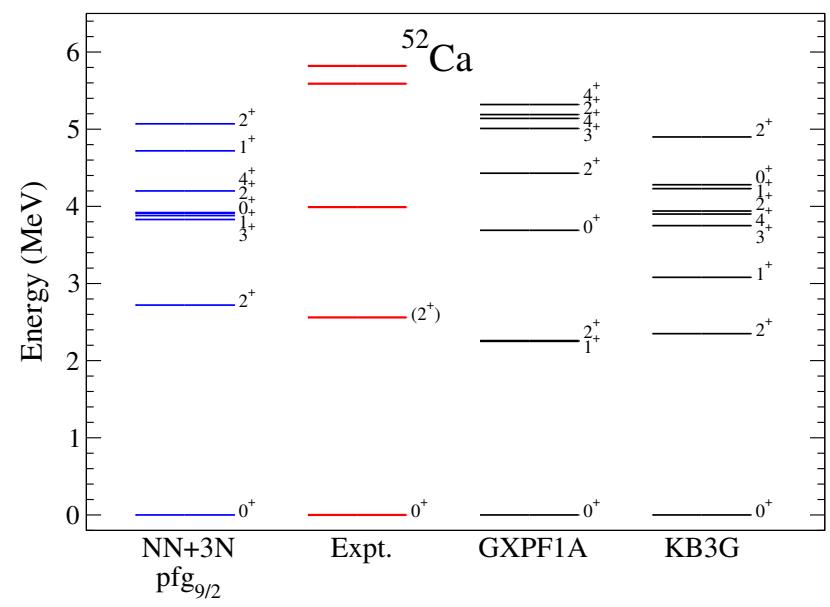

FIG. 11. (Color online) Excitation energies of bound excited states in ${ }^{52} \mathrm{Ca}$ compared with experiment [14, 17] and phenomenological interactions (labels as in Fig. 6.

lying $1 \mathrm{MeV}$ higher for $\mathrm{KB} 3 \mathrm{G}$ and $2 \mathrm{MeV}$ higher for GXPF1A. The reason for the difference when using $3 \mathrm{~N}$ forces is related to the low $5 / 2^{-}$state in ${ }^{49} \mathrm{Ca}$, originating from the small effective $p_{1 / 2}-f_{5 / 2}$ gap in our MBPT approach. Note that this effective gap is also significantly different between the phenomenological interactions. In turn, the $5 / 2_{2}^{-}$state in the MBPT calculations has a $\left(p_{3 / 2}\right)_{J=2}^{2}\left(p_{1 / 2}\right)^{1}$ dominant structure (on top of ${ }^{48} \mathrm{Ca}$ ) and is therefore related to the $2_{1}^{+}$state in ${ }^{50} \mathrm{Ca}$. In all calculations it agrees with the tentatively assigned experimental state at $2.4 \mathrm{MeV}$. Ultimately, improved gamma-ray spectroscopy is needed. 


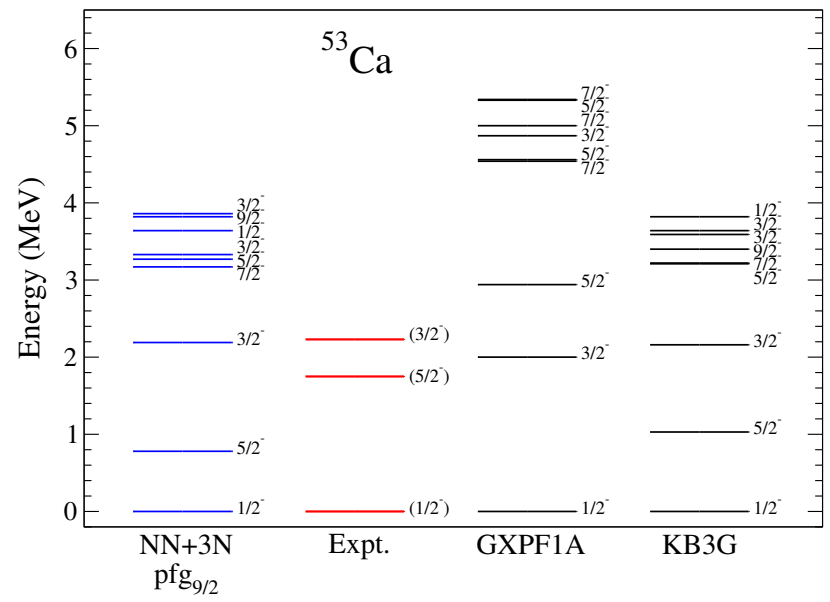

FIG. 12. (Color online) Excitation energies of bound excited states in ${ }^{53} \mathrm{Ca}$ compared with experiment [15] and phenomenological interactions (labels as in Fig. 6.

6. ${ }^{52} \mathrm{Ca}$

For ${ }^{52} \mathrm{Ca}$, there are no spin assignments except for the ground and first-excited state, where the large spacing was first identified as a signature of the $N=32$ subshell closure [3. The strong $N=32$ shell closure has been unambiguously established with mass measurements out to ${ }^{54} \mathrm{Ca}$, leading to a steep decrease of the two-neutron separation energy after ${ }^{52} \mathrm{Ca}$ [10. In Fig. 11 our NN+3N calculations are compared to the phenomenological interactions. All agree well with the limited experimental data.

One striking difference between models, however, is the location of the $1_{1}^{+}$state, which is found in our MBPT calculations $1 \mathrm{MeV}$ and $2 \mathrm{MeV}$ above the KB3G and GXPF1A calculations, respectively, and hence an accurate experimental measurement would be highly valuable. The $3_{1}^{+}$state is also predicted quite differently depending on the calculation; our MBPT value is in good agreement with KB3G but more than $1 \mathrm{MeV}$ below that of GXPF1A.

$$
\text { 7. }{ }^{53} \mathrm{Ca}
$$

Only the ground-state spin of ${ }^{53} \mathrm{Ca}$ and the position of two excited states are known experimentally, one of them only measured very recently at RIKEN [15]. Figure 12 shows our $\mathrm{NN}+3 \mathrm{~N}$ calculations in the $p f g_{9 / 2}$ space compared to the phenomenological interactions. In this spectrum the ground state is dominated by a $p_{1 / 2}$ hole in the $N=34$ closed subshell. Therefore, the difference between the ground and first $5 / 2_{1}^{-}$and $3 / 2_{1}^{-}$states will be related to the effective $p_{1 / 2}-f_{5 / 2}$ and $p_{1 / 2}-p_{3 / 2}$ gaps and hence the strengths of the $N=32$ and $N=34$ subshell closures, respectively. All three calculations predict a consistent location for the lowest $3 / 2_{1}^{-}$state, also

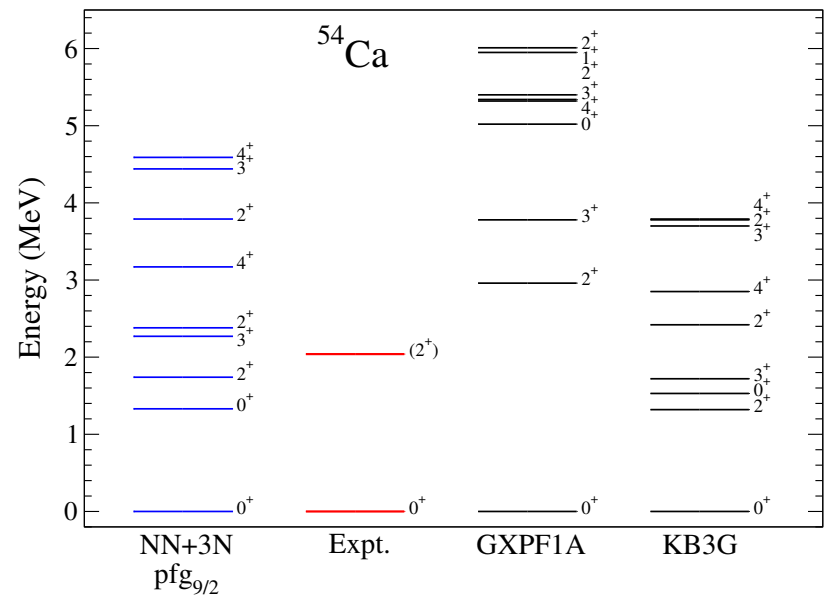

FIG. 13. (Color online) Excitation energies of bound excited states in ${ }^{54} \mathrm{Ca}$ compared with experiment [15] and phenomenological interactions (labels as in Fig. 6).

in agreement with the unassigned experimental state at $2.2 \mathrm{MeV}$, which, assuming this is the correct spin assignment, reflects the predictions of the $2_{1}^{+}$state in ${ }^{52} \mathrm{Ca}$.

Interestingly, the $5 / 2_{1}^{-}$state appears at different excitation energies in all calculations: $0.8 \mathrm{MeV}$ in MBPT, $1 \mathrm{MeV}$ with KB3G, and $3 \mathrm{MeV}$ with GXPF1A, in comparison with the state with unassigned spin at $1.75 \mathrm{MeV}$. This shows that phenomenological interactions, which give similar results close to stability, can extrapolate to very different results for neutron-rich systems. In this case, the difference is related to the small $p_{1 / 2}-f_{5 / 2}$ gap (weak $N=34$ subsell closure) predicted by KB3G, and also preferred by our MBPT approach, in contrast with the large gap (strong $N=34$ subsell closure) given by GXPF1A. Improved versions of GXPF1A that adjust the $p_{1 / 2}^{2}$ and $p_{1 / 2}-f_{5 / 2} T=1$ monopole matrix elements according to the most recent experimental data, GXPF1B and modifications, have recently become available [15, 23]. They reduce the $p_{1 / 2}-f_{5 / 2}$ gap in agreement with experiment, and predict the $5 / 2_{1}^{-}$state at $1.9 \mathrm{MeV}$. The two excited states are also in good agreement with recent $\mathrm{CC}$ calculations with phenomenological $3 \mathrm{~N}$ forces, which predict the $5 / 2_{1}^{-}$and $3 / 2_{1}^{-}$states at 1.9 and $2.5 \mathrm{MeV}$, respectively 33 .

$$
\text { 8. }{ }^{54} \mathrm{Ca}
$$

${ }^{54} \mathrm{Ca}$ is the last calcium isotope for which spectroscopic data exists. In Fig. 13 we show our $\mathrm{NN}+3 \mathrm{~N}$ calculations compared with the phenomenological interactions and the recent breakthrough $2_{1}^{+}$measurement at $2.043(19) \mathrm{MeV}$ [15. Our MBPT calculations predict several low-lying excited states, implying only a weak $N=34$ subshell closure, consistent with the spectrum in ${ }^{53} \mathrm{Ca}$. In particular, the $2_{1}^{+}$state is predicted at $1.7 \mathrm{MeV}$ [32, only $300 \mathrm{keV}$ below experiment. The $2_{1}^{+}$ 


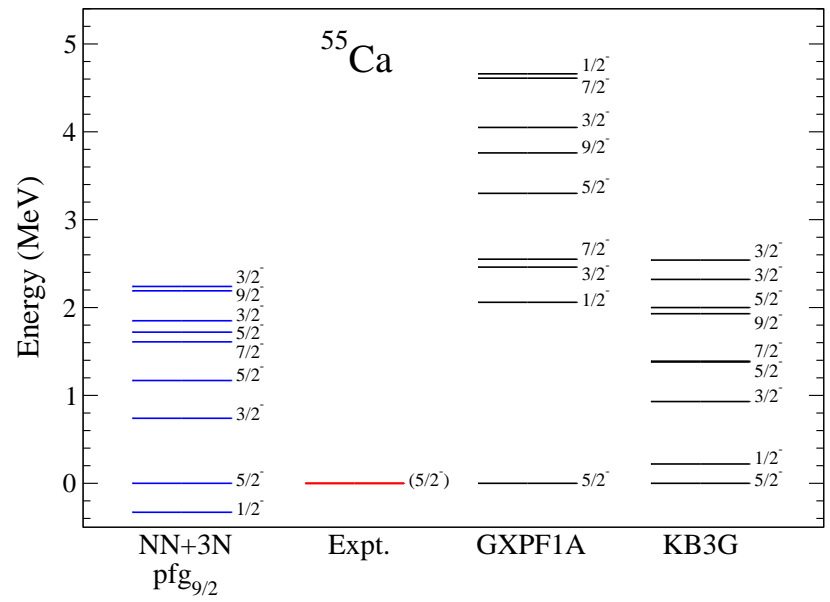

FIG. 14. (Color online) Excitation energies in ${ }^{55} \mathrm{Ca}$ compared with phenomenological interactions (labels as in Fig. 6).

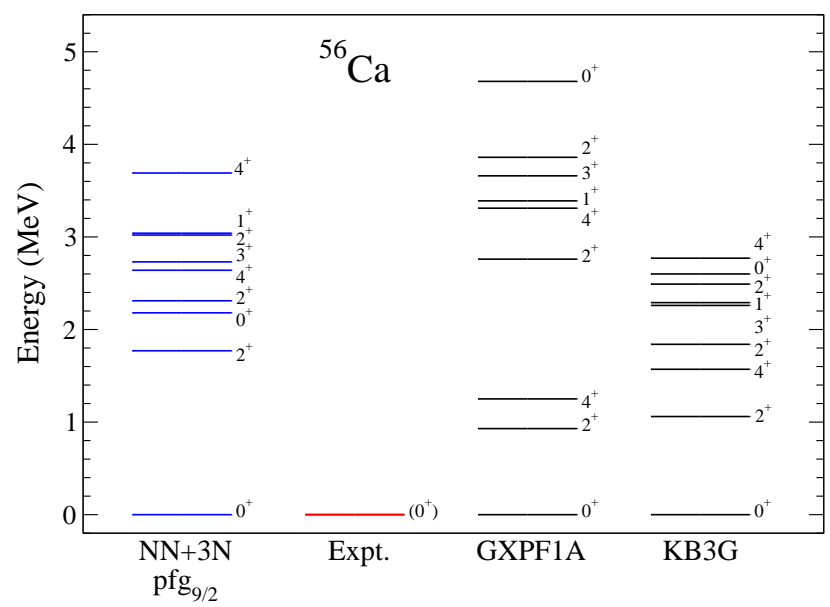

FIG. 15. (Color online) Excitation energies in ${ }^{56} \mathrm{Ca}$ compared with phenomenological interactions (labels as in Fig. 6).

excitation energy is also in very good agreement with 1.9 MeV predicted by CC calculations with phenomenological $3 \mathrm{~N}$ forces 33 .

The striking difference between KB3G and GXPF1A in this region is clearly manifested in ${ }^{54} \mathrm{Ca}$. The recently measured $2_{1}^{+}$energy lies $1 \mathrm{MeV}$ below GXPF1A and $0.7 \mathrm{MeV}$ above the KB3G prediction. The difference between these calculations is consistent with the spectra presented in the discussion of ${ }^{53} \mathrm{Ca}$. As in ${ }^{53} \mathrm{Ca}$, this is improved when considering the modified GXPF1B interaction, which reproduces experiment [15].

$$
\text { 9. }{ }^{55} \mathrm{Ca} \text { and }{ }^{56} \mathrm{Ca}
$$

Finally, in Figs. 14 and 15 we show NN+3N predictions for the spectra in ${ }^{55} \mathrm{Ca}$ and ${ }^{56} \mathrm{Ca}$. In this region the importance of the neutron $1 d_{5 / 2}$ and $2 s_{1 / 2}$ orbitals, currently not included in our calculations, has been empha-

\begin{tabular}{ccccc}
\hline \hline Transition & KB3G & GXPF1A & MBPT & Exp. \\
\hline${ }^{46} \mathrm{Ca}: 2^{+} \rightarrow 0^{+}$ & 9.2 & 9.2 & 13.3 & $25.4 \pm 4.5$ \\
& & & & $36.4 \pm 2.6$ \\
${ }^{46} \mathrm{Ca}: 4^{+} \rightarrow 2^{+}$ & 7.5 & 7.1 & 9.9 & $8.6 \pm 2.1$ \\
${ }^{46} \mathrm{Ca}: 6^{+} \rightarrow 4^{+}$ & 3.6 & 3.6 & 4.8 & $5.38 \pm 0.29$ \\
${ }^{47} \mathrm{Ca}: 3 / 2^{-} \rightarrow 7 / 2^{-}$ & 0.84 & 3.6 & 1.0 & $4.0 \pm 0.2$ \\
${ }^{48} \mathrm{Ca}: 2^{+} \rightarrow 0^{+}$ & 11.5 & 11.9 & 10.3 & $19 \pm 6.4$ \\
${ }^{49} \mathrm{Ca}: 7 / 2^{-} \rightarrow 3 / 2^{-}$ & 0.41 & 4.0 & 0.22 & $0.53 \pm 0.21$ \\
${ }^{50} \mathrm{Ca}: 2^{+} \rightarrow 0^{+}$ & 8.9 & 9.1 & 11.2 & $7.4 \pm 0.2$ \\
\hline \hline
\end{tabular}

TABLE II. Electric quadrupole transition rates in the calcium isotopes compared with experiment $[18,64$. The $B(E 2)$ values are calculated in the $p f g_{9 / 2}$ space with $\mathrm{NN}+3 \mathrm{~N}$ interactions. Effective charges $\delta q=0.5 e$ are used in all calculations, which employ a harmonic-oscillator length $b=(\hbar / m \omega)^{1 / 2}$ with $\hbar \omega=\left(45 A^{-1 / 3}-25 A^{-2 / 3}\right) \mathrm{MeV}$ and nucleon mass $m$. The units are $e^{2} \mathrm{fm}^{4}$.

sized in Refs. 33, 63. We plan to extend our approach to include these orbitals in the valence space and provide an improved description of very neutron-rich nuclei near $N=40$.

Our $\mathrm{NN}+3 \mathrm{~N}$ prediction for ${ }^{55} \mathrm{Ca}$ in Fig. 14 largely agrees with the predictions of the KB3G interaction, with a number of close-lying states below $2.5 \mathrm{MeV}$, in contrast to GXPF1A, where the first excited state lies above $2 \mathrm{MeV}$. However, we find a $1 / 2^{-}$ground state, rather than $5 / 2^{-}$as predicted in KB3G, GXPF1A and the CC calculations of Ref. 33. This is consistent with the picture of a weak $N=34$ shell closure in ${ }^{54} \mathrm{Ca}$, where states in ${ }^{55} \mathrm{Ca}$ are not dominated by single-particle character.

In ${ }^{56} \mathrm{Ca}$ the $\mathrm{NN}+3 \mathrm{~N} 2{ }_{1}^{+}$state is around $800 \mathrm{keV}$ higher than in KB3G, GXPF1A and CC 33, the same trend of higher $2_{1}^{+}$states than in phenomenological interactions for non-closed even-even calcium isotopes. Otherwise the spacing of excited states in our $\mathrm{NN}+3 \mathrm{~N}$ calculation is closer to KB3G. The differences between all three interactions in Fig. 15 highlight the importance of experimental spectroscopic studies beyond ${ }^{54} \mathrm{Ca}$.

\section{Electromagnetic transition strengths}

$$
\text { 1. } B(E 2)
$$

Next we study electromagnetic quadrupole $(E 2)$ transitions. The results of our theoretical calculations are compared with experiment [18, 64, in Table II and with those of the phenomenological interactions. Standard effective charges $\delta q=0.5 e$, which take into account the reduced valence space of the calculations, are employed in all cases. Work is in progress on calculating the consistent effective one-body operators within MBPT.

Overall, we find good agreement between our valencespace calculations and experiment. The measured $B(E 2)$ transitions in Table II vary widely (within a factor of 50), therefore obtaining agreement within experimental error bars is particularly challenging. Indeed the calculated 


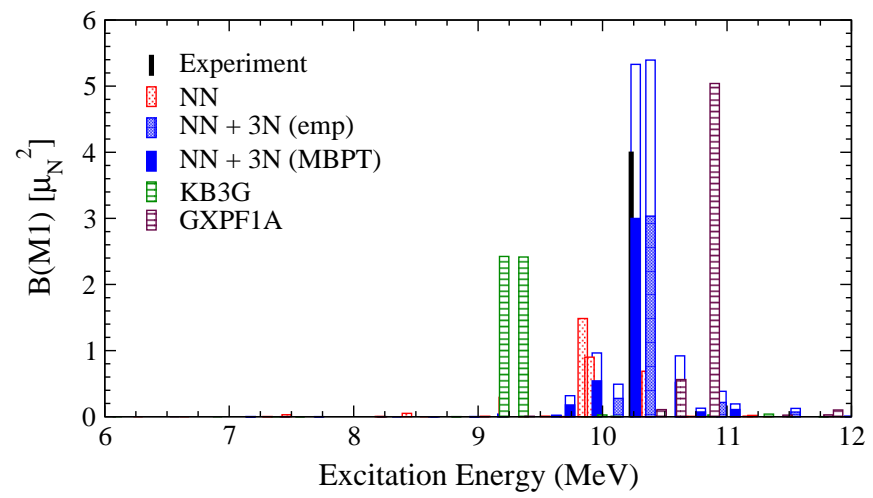

FIG. 16. (Color online) Magnetic dipole transition rates from the ground state to $1^{+}$excited states in ${ }^{48} \mathrm{Ca}$ compared with experiment 67. The $B(M 1)$ values are calculated in the $p f g_{9 / 2}$ space with $\mathrm{NN}+3 \mathrm{~N}$ interactions. Spin $g$ factors are quenched by 0.75 , except for the empty blue bars.

$B(E 2)$ values can deviate from experiment by up to a factor of two (or a factor of four for the small $3 / 2^{-} \rightarrow 7 / 2^{-}$ transition in ${ }^{47} \mathrm{Ca}$ ), which is similar to the phenomenological interactions.

In transitions involving ${ }^{46} \mathrm{Ca}$, the MBPT $B(E 2)$ results are systematically $30 \%$ larger than with the phenomenological interactions and lie closer to experiment. Nevertheless the predicted $2^{+} \rightarrow 0^{+}$transition in ${ }^{46} \mathrm{Ca}$ is still half the experimental value, pointing to important missing contributions (we also note that the two different experimental values do not overlap). In transitions involving ${ }^{47} \mathrm{Ca},{ }^{48} \mathrm{Ca}$, and ${ }^{49} \mathrm{Ca}$, the MBPT $B(E 2)$ values are similar to those obtained with $\mathrm{KB} 3 \mathrm{G}$, but all calculations underestimate experiment. This also applies to GXPF1A for the $2^{+} \rightarrow 0^{+}$transition in ${ }^{48} \mathrm{Ca}$. For the $3 / 2^{-} \rightarrow 7 / 2^{-}$ transition in ${ }^{47} \mathrm{Ca}$, GXPF1A finds very good agreement with data, with the caveat that the $7 / 2^{-} \rightarrow 3 / 2^{-}$transition in ${ }^{49} \mathrm{Ca}$ is overpredicted. The MBPT $B(E 2)$ values for the $2^{+} \rightarrow 0^{+}$transition in ${ }^{50} \mathrm{Ca}$ are $20 \%$ larger than with the phenomenological interactions, in this case $50 \%$ higher than experiment.

Finally, we emphasize that electroweak two-body currents (meson-exchange currents) have not been included in our calculations of electromagnetic transitions. These have been derived consistently in chiral EFT and shown to be important for magnetic moments and electromagnetic transitions in light nuclei 65. For axial-vector weak interactions, chiral two-body currents have been applied to medium-mass nuclei, showing that they provide important contributions to the quenching of Gamow-Teller transitions 66. Work is in progress to extend this to electromagnetic currents and to calculate effective operators consistently in MBPT.

\section{2. $B(M 1)$}

The magnetic dipole $(M 1)$ transition between the ground state of ${ }^{48} \mathrm{Ca}$ and $1^{+}$excited states is compared to experiment [67] in Fig. 16. All results use spin $g$ factors with empirical quenching $q=0.75$, except for the empty blue bars, which give the $\mathrm{NN}+3 \mathrm{~N}$ results without quenching. The main conclusions of previous work using $3 \mathrm{~N}$ forces to first order in MBPT still apply [28. With NN forces only, the $B(M 1)$ strength is strongly fragmented with a central value well below experiment. For the phenomenological interactions, GXPF1A finds a concentrated peak, while for KB3G the total strength is evenly fragmented between two peaks. We note, however, that the related KB3 interaction [68] finds no fragmentation, which highlights the particular sensitivity of this transition to the valence-space Hamiltonian. The peaks lie about $1 \mathrm{MeV}$ lower than experiment for KB3G, and around $700 \mathrm{keV}$ higher for GXPF1A. When 3N forces are included in the $p f g_{9 / 2}$ space, agreement with experimental data is clearly improved, with a concentrated peak very close to the experimental value. The effects of the improved treatment of $3 \mathrm{~N}$ forces over that in Ref. [28] is an increase of the excitation energy of the peak transition by $\sim 500 \mathrm{keV}$. The degree of single-particle character of the $M 1$ transition, once $3 \mathrm{~N}$ forces are included, is similar for calculated (MBPT) or empirical (emp) SPEs. Finally, it is interesting that the $\mathrm{NN}+3 \mathrm{~N}$ calculations do not require a strong quenching of spin $g$ factors. While the unquenched $\mathrm{NN}+3 \mathrm{~N}$ results indeed overpredict the $M 1$ transition strength, they are already at the level of the quenched GXPF1A value; only a modest quenching of 0.9 would be needed to reproduce the experimental strength.

\section{Residual $\mathbf{3 N}$ forces}

We have also explored residual $3 \mathrm{~N}$ forces, which arise between three valence nucleons in addition to their oneand two-body normal-ordered contributions. Note that in the shell model the normal ordering is performed with respect to the ${ }^{40} \mathrm{Ca}$ core. This does not take into account the contributions from MBPT outside of the valence space. In general, residual $3 \mathrm{~N}$ forces are expected to lead to small corrections due to phase-space considerations for normal Fermi systems [53], because their effects are suppressed by the number of valence particles to the number of particles in the core. We have calculated the contributions from residual $3 \mathrm{~N}$ forces at first order in perturbation theory, using the states obtained from $\mathrm{NN}+3 \mathrm{~N}$ interactions at the normal-ordered one- and twobody level (as discussed in the previous sections).

In Fig. 17 we show the resulting energies $\Delta E_{3 \mathrm{~N} \text {,res }}=$ $\left\langle\psi_{n}\left|V_{\text {res }}^{3 \mathrm{~N}}\right| \psi_{n}\right\rangle$ for the ground state and first excited state calculated in the extended $p f g_{9 / 2}$ space. As expected from neutron-matter calculations 69, 70, residual 3N forces are repulsive. Their role is very minor for the 


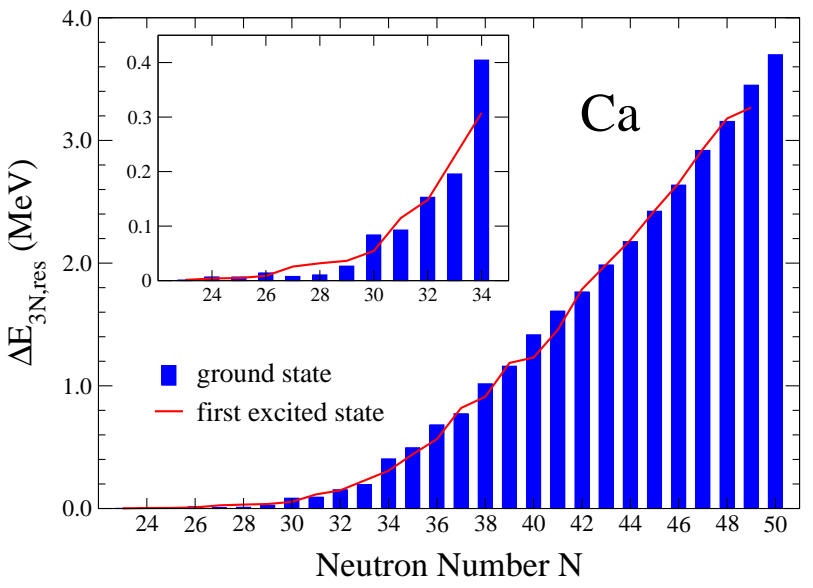

FIG. 17. (Color online) First-order contribution from residual $3 \mathrm{~N}$ forces to the energies of the ground state (bars) and first excited state (solid line) of the calcium isotopes in the extended $p f g_{9 / 2}$ valence space.

lighter isotopes, but their contribution is amplified with neutron number, increasing to a maximum contribution of $3.7 \mathrm{MeV}$ in ${ }^{70} \mathrm{Ca}$. For the mid shell $A=55-58$ isotopes, only a subset of residual $3 \mathrm{~N}$ matrix elements was used. From tests in other isotopes, we estimate the difference to using the full set to be less than $5 \%$ of the total residual $3 \mathrm{~N}$ energy contribution.

We compare the effects of residual $3 \mathrm{~N}$ forces with the $\mathrm{NN}+3 \mathrm{~N}$ ground-state energies of Fig. 4 at the normalordered two-body level. This shows that residual $3 \mathrm{~N}$ forces provide small corrections to the ground-state energy, ranging from at most $3 \%$ (in ${ }^{56} \mathrm{Ca}$ ) for the isotopes discussed in Sect. III B to $9 \%$ in ${ }^{70} \mathrm{Ca}$. This justifies our perturbative estimate of residual $3 \mathrm{~N}$ forces. As shown in Fig. 17, residual 3N contributions are similar for excited states as for ground states. Therefore, residual $3 \mathrm{~N}$ forces lead to even smaller corrections for excitation energies, of $20 \mathrm{keV}$ for ${ }^{48} \mathrm{Ca}$, increasing to $100 \mathrm{keV}$ in ${ }^{54} \mathrm{Ca}$, and never exceeding $200 \mathrm{keV}$. As a result, the uncertainty from not including residual $3 \mathrm{~N}$ forces is small for the results presented in the previous sections.

\section{SUMMARY}

We have presented a comprehensive study of excitedstate properties of calcium isotopes based on chiral $\mathrm{NN}+3 \mathrm{~N}$ interactions. The theoretical approach has been discussed in detail, focusing on convergence properties in the MBPT framework, benchmarking against ab initio $\mathrm{CC}$ theory for NN interactions, and exploring the role of residual $3 \mathrm{~N}$ forces. We have presented results for groundstate energies and spectra for neutron-rich isotopes to ${ }^{56} \mathrm{Ca}$, where $3 \mathrm{~N}$ forces were shown to be key to understand the experimental structures. With both $3 \mathrm{~N}$ forces and an extended $p f g_{9 / 2}$ valence space, we obtain a good level of agreement with experiment, where the extended

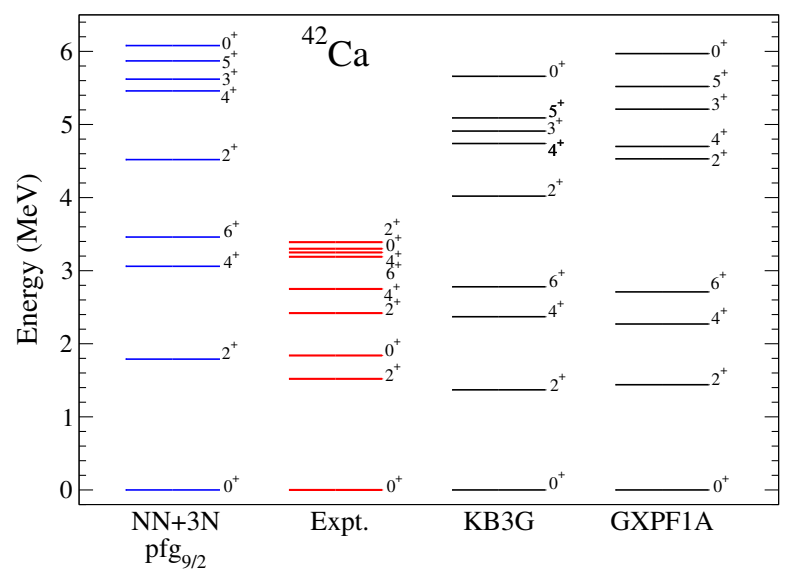

FIG. 18. (Color online) Excitation energies of bound excited states in ${ }^{42} \mathrm{Ca}$ compared with experiment and phenomenological interactions (labels as in Fig. 6.

space is especially important for $N \geqslant 28$. We have also studied electromagnetic $E 2$ and $M 1$ transitions, finding that experimental data are well described by our calculations. Where data does not exist, our results provide predictions for unexplored properties of neutron-rich calcium isotopes.

Future work will include studies of the theoretical uncertainties due to the input Hamiltonian and the RG or SRG evolution. In addition, the recent development of the IMSRG for open shell [71, 72] and CC calculations of effective interactions 73 . enable nonperturbative derivations of valence-space Hamiltonians, which will also provide benchmarks for MBPT calculations.

\section{ACKNOWLEDGMENTS}

We thank G. Hagen for helpful discussions. This work was supported by the BMBF Contract No. 06DA70471, the DFG through Grant SFB 634, the ERC Grant No. 307986 STRONGINT, and the Helmholtz Alliance HA216/EMMI. Computations were performed on JUROPA at the Jülich Supercomputing Center and on Kraken at the National Institute for Computational Sciences.

\section{Appendix A: Spectra of light calcium isotopes $A<47$}

We present the spectra for the lighter ${ }^{42-46} \mathrm{Ca}$ in Figs. 1822 . Our results are in good agreement with experiment [62], and generally exhibit quality comparable to phenomenological interactions. The only exceptions are the $4^{+}, 6^{+}$states in ${ }^{46} \mathrm{Ca}, \sim 1 \mathrm{MeV}$ higher than in experiment, in contrast to phenomenology. Note that some excited states, like the $0_{2}^{+}$states in ${ }^{42,44,46} \mathrm{Ca}$, are expected to be dominated by $s d$ shell degrees of freedom, and are therefore not present in our theoretical results. 


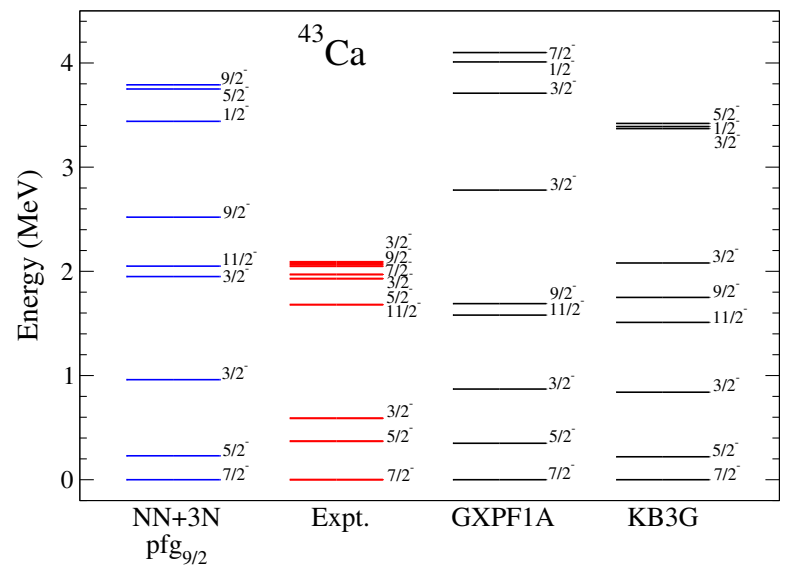

FIG. 19. (Color online) Excitation energies of bound excited states in ${ }^{43} \mathrm{Ca}$ compared with experiment and phenomenological interactions (labels as in Fig. 6).

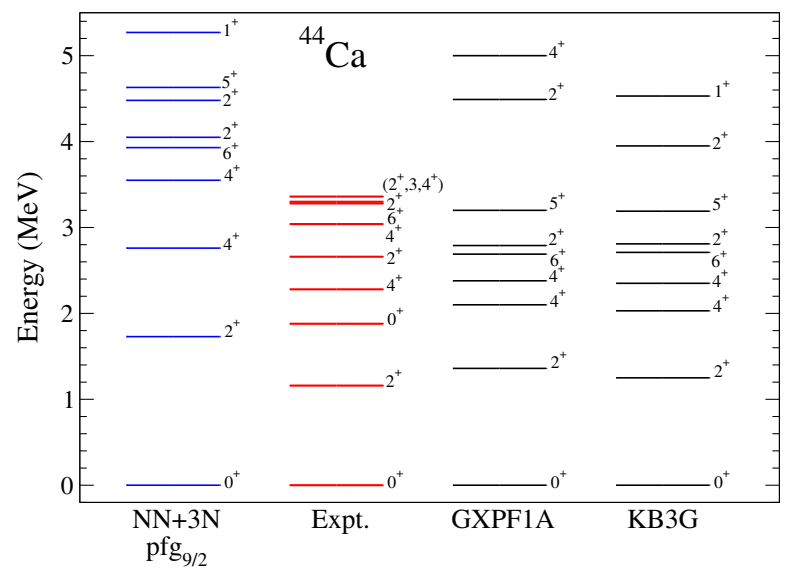

FIG. 20. (Color online) Excitation energies of bound excited states in ${ }^{44} \mathrm{Ca}$ compared with experiment and phenomenological interactions (labels as in Fig. 6).

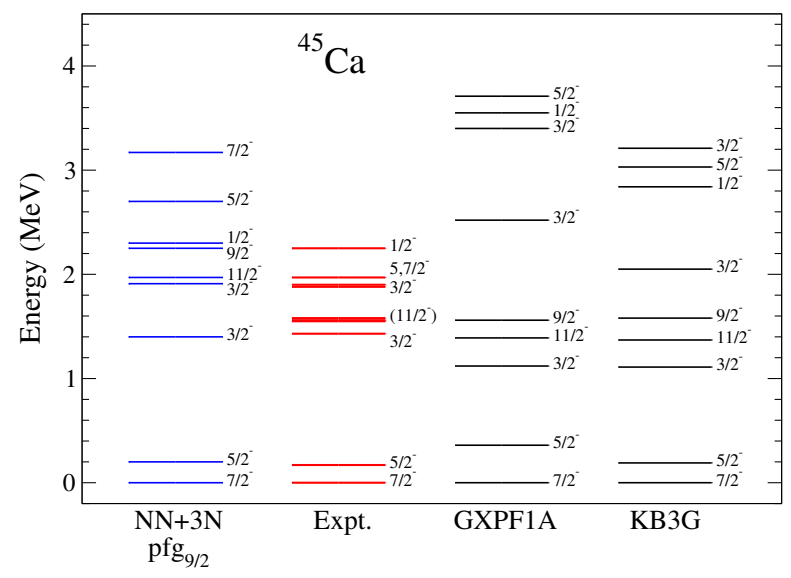

FIG. 21. (Color online) Excitation energies of bound excited states in ${ }^{45} \mathrm{Ca}$ compared with experiment and phenomenological interactions (labels as in Fig. 6.

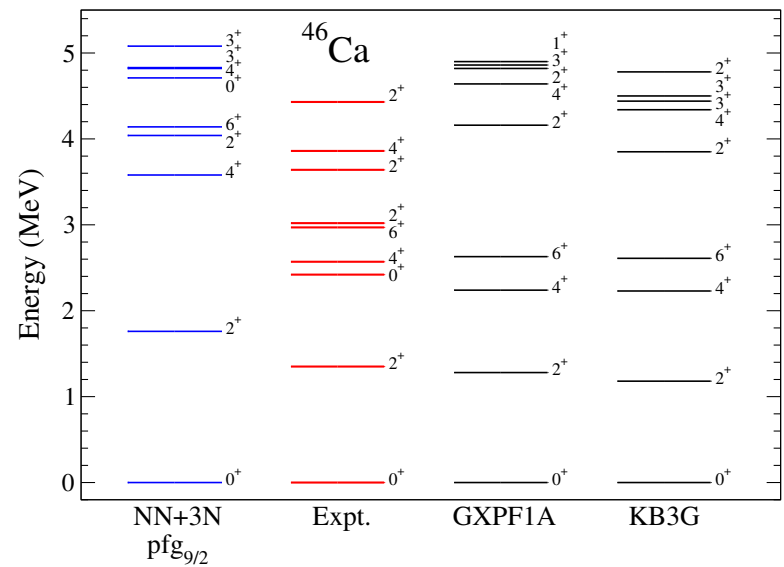

FIG. 22. (Color online) Excitation energies of bound excited states in ${ }^{46} \mathrm{Ca}$ compared with experiment and phenomenological interactions (labels as in Fig. 6.

[6] P. F. Mantica, R. Broda, H. L. Crawford, A. Damaske, B. Fornal, A. A. Hecht, C. Hoffman, M. Horoi, N. Hoteling, R. V. F. Janssens et al., Phys. Rev. C 77, 014313 (2008).

[7] H. L. Crawford, R. V. F. Janssens, P. F. Mantica, J. S. Berryman, R. Broda, M. P. Carpenter, N. Cieplicka, B. Fornal, G. F. Grinyer, N. Hoteling et al., Phys. Rev. C 82, 014311 (2010).

[8] J. I. Prisciandaro, P. F. Mantica, B. A. Brown, D. W. Anthony, M. W. Cooper, A. Garcia, D. E. Groh, A. Komives, W. Kumarasiri, P. A. Lofy et al., Phys. Lett. B 510, 17 (2001).

[9] A. T. Gallant, J. C. Bale, T. Brunner, U. Chowdhury, S. Ettenauer, A. Lennarz, D. Robertson, V. V. Simon, A. Chaudhuri, J. D. Holt et al., Phys. Rev. Lett. 109, 032506 (2012). 
[10] F. Wienholtz, D. Beck, K. Blaum, Ch. Borgmann, M. Breitenfeldt, R. B. Cakirli, S. George, F. Herfurth, J. D. Holt, M. Kowalska et al., Nature 498, 346 (2013).

[11] N. Marginean, S. M. Lenzi, A. Gadea, E. Farnea, S. J. Freeman, D. R. Napoli, D. Bazzacco, S. Beghini, B. R. Behera, P. G. Bizzeti et al., Phys. Lett. B 633, 696 (2006).

[12] S. N. Liddick, P. F. Mantica, R. V. F. Janssens, R. Broda, B. A. Brown, M. P. Carpenter, B. Fornal, M. Honma, T. Mizusaki, A. C. Morton et al., Phys. Rev. Lett. 92, 072502 (2004).

[13] D.-C. Dinca, R. V. F. Janssens, A. Gade, D. Bazin, R. Broda, B. A. Brown, C. M. Campbell, M. P. Carpenter, P. Chowdhury, J. M. Cook et al., Phys. Rev. C 71, 041302 (2005).

[14] M. Rejmund, S. Bhattacharyya, A. Navin, W. Mittig, L. Gaudefroy, M. Gelin, G. Mukherjee, F. Rejmund, P. Roussel-Chomaz, and Ch. Theisen, Phys. Rev. C 76, 021304(R) (2007).

[15] D. Steppenbeck, S. Takeuchi, N. Aoi, P. Doornenbal, M. Matsushita, H. Wang, H. Baba, N. Fukuda, S. Go, M. Honma et al., Nature 502, 207 (2013).

[16] F. Perrot, F. Maréchal, C. Jollet, Ph. Dessagne, J.C. Angélique, G. Ban, P. Baumann, F. Benrachi, U. Bergmann, C. Borcea et al., Phys. Rev. C 74, 014313 (2006).

[17] B. Fornal, R. V. F. Janssens, R. Broda, N. Marginean, S. Beghini, L. Corradi, M. P. Carpenter, G. De Angelis, F. Della Vedova, E. Farnea et al., Phys. Rev. C 77, 014304 (2008).

[18] D. Montanari, S. Leoni, D. Mengoni, J. J. ValienteDobon, G. Benzoni, N. Blasi, G. Bocchi, P. F. Bortignon, S. Bottoni, A. Bracco et al., Phys. Rev. C 85, 044301 (2012).

[19] E. Caurier, G. Martinez-Pinedo, F. Nowacki, A. Poves, A. P. Zuker, Rev. Mod. Phys. 77, 427(2005).

[20] A. Poves, J. Sánchez-Solano, E. Caurier, and F. Nowacki, Nucl. Phys. A 694, 157 (2001).

[21] M. T. Honma, T. Otsuka, B. A. Brown, and T. Mizusaki, Phys. Rev. C 69, 034335 (2004).

[22] M. T. Honma, T. Otsuka, B. A. Brown, and T. Mizusaki, Eur. J. Phys. A 25, 499 (2005).

[23] Y. Utsuno, T. Otsuka, B. A. Brown, M. T. Honma, T. Mizusaki, and N. Shimizu, Phys. Rev. C 86, 051301(R) (2012).

[24] T. R. Rodriguez and J. L. Egido, Phys. Rev. Lett. 99, 062501 (2007).

[25] M. Hjorth-Jensen, T. T. S. Kuo, and E. Osnes, Phys. Rept. 261, 125 (1995).

[26] A. P. Zuker, Phys. Rev. Lett. 90, 042502 (2003).

[27] H.-W. Hammer, A. Nogga, and A. Schwenk, Rev. Mod. Phys. 85, 197 (2013).

[28] J. D. Holt, T. Otsuka, A. Schwenk, and T. Suzuki, J. Phys. G 39, 085111 (2012).

[29] T. Otsuka, T. Suzuki, J. D. Holt, A. Schwenk, and Y. Akaishi, Phys. Rev. Lett. 105, 032501 (2010).

[30] J. D. Holt, J. Menéndez, and A. Schwenk, Eur. Phys. J. A 49, 39 (2013).

[31] J. D. Holt, J. Menéndez, and A. Schwenk, Phys. Rev. Lett. 110, 022502 (2013).

[32] J. D. Holt, J. Menéndez, and A. Schwenk, J. Phys. G 40, 075105 (2013).

[33] G. Hagen, M. Hjorth-Jensen, G. R. Jansen, R. Machleidt, and T. Papenbrock, Phys. Rev. Lett. 109, 032502 (2012).
[34] V. Somà, C. Barbieri, and T. Duguet, Phys. Rev. C 87, 011303 (2013).

[35] V. Somà, A. Cipollone, C. Barbieri, P. Navrátil, and T. Duguet, arXiv:1312.2068.

[36] R. Roth, S. Binder, K. Vobig, A. Calci, J. Langhammer, and P. Navrátil, Phys. Rev. Lett. 109, 052501 (2012).

[37] K. Tsukiyama, S. K. Bogner, and A. Schwenk, Phys. Rev. Lett. 106, 222502 (2011).

[38] H. Hergert, S. K. Bogner, S. Binder, A. Calci, J. Langhammer, R. Roth, and A. Schwenk, Phys. Rev. C 87, 034307 (2013).

[39] E. Epelbaum, H.-W. Hammer and U.-G. Meißner, Rev. Mod. Phys. 81, 1773 (2009).

[40] R. Machleidt and D. Entem, Phys. Rept. 503, 1 (2011).

[41] C. Caesar, J. Simonis, T. Adachi, Y. Aksyutina, J. Alcantara, S. Altstadt, H. Alvarez-Pol, N. Ashwood, T. Aumann, V. Avdeichikov et al., Phys. Rev. C 88, 034313 (2013).

[42] G. Hagen, T. Papenbrock, M. Hjorth-Jensen, and D. J. Dean, arXiv:1312.7872

[43] T. T. S. Kuo and E. Osnes, Springer Lecture Notes of Physics, Vol. 364, p. 1 (1990).

[44] S. K. Bogner, T. T. S. Kuo, L. Coraggio, A. Covello, and N. Itaco, Phys. Rev. C 65, 051301(R) (2002).

[45] L. Coraggio, A. Covello, A. Gargano, and N. Itaco, Phys. Rev. C 80, 044311 (2009).

[46] S. K. Bogner, T. T. S. Kuo, and A. Schwenk, Phys. Rept. 386, 1 (2003)

[47] S. K. Bogner, R. J. Furnstahl, and A. Schwenk, Prog. Part. Nucl. Phys. 65, 94 (2010).

[48] D. R. Entem and R. Machleidt, Phys. Rev. C 68, 041001(R) (2003).

[49] S. K. Bogner, R. J. Furnstahl, S. Ramanan, and A. Schwenk, Nucl. Phys. A 784, 79 (2007).

[50] U. van Kolck, Phys. Rev. C 49, 2932 (1994).

[51] E. Epelbaum, A. Nogga, W. Glöckle, H. Kamada, U.-G. Meißner, and H. Witała, Phys. Rev. C 66, 064001 (2002).

[52] S. K. Bogner, R. J. Furnstahl, A. Nogga, and A. Schwenk, arXiv:0903.3366 (2009); K. Hebeler, S. K. Bogner, R. J. Furnstahl, A. Nogga, and A. Schwenk, Phys. Rev. C 83, 031301(R) (2010).

[53] B. Friman and A. Schwenk, in From Nuclei to Stars: Festschrift in Honor of Gerald E. Brown, Ed. S. Lee (World Scientific, 2011) arXiv:1101.4858.

[54] G. Hagen, D. J. Dean, M. Hjorth-Jensen, T. Papenbrock, and A. Schwenk, Phys. Rev. C 76, 034302 (2007).

[55] G. Hagen, T. Papenbrock, D. J. Dean, and M. HjorthJensen, Phys. Rev. C 82, 034330 (2010).

[56] E. Caurier and F. Nowacki, Acta Phys. Pol. 30, 705 (1999).

[57] D. J. Dean, M. T. Ressell, M. Hjorth-Jensen, S. E. Koonin, K. Langanke, and A. P. Zuker, Phys. Rev. C 59, 2474 (1999).

[58] E. Caurier, J. Menéndez, F. Nowacki, and A. Poves, Phys. Rev. C 75, 054317 (2007).

[59] G. Audi, M. Wang, A. H. Wapstra, F. G. Kondev, M. MacCormick, X. Xu, and B. Pfeiffer, Chin. Phys C 36, 1287 (2012).

[60] S. Okubo, Prog. Theor. Phys. 12, 603 (1954).

[61] S. Y. Lee and K. Suzuki, Phys. Lett. B 91, 173 (1980).

[62] http://www.nndc.bnl.gov/ensdf/

[63] S. M. Lenzi, F. Nowacki, A. Poves, and K. Sieja, Phys. Rev. C 82, 054301 (2010). 
[64] S. Raman, C. W. G. Nestor, Jr. and P. Tikkanen, At. Data Nucl. Data Tables 78, 1 (2001).

[65] S. Pastore, S. C. Pieper, R. Schiavilla, and R. B. Wiringa, Phys. Rev. C 87, 035503 (2013).

[66] J. Menéndez, D. Gazit, and A. Schwenk, Phys. Rev. Lett. 107, 062501 (2011).

[67] P. von Neumann-Cosel, A. Poves, J. Retamosa, and A. Richter, Phys. Lett. B 443, 1 (1998).

[68] A. Poves and A. P. Zuker, Phys. Rep. 70, 235 (1980).
[69] K. Hebeler and A. Schwenk, Phys. Rev. C 82, 014314 (2010).

[70] K. Hebeler and A. Schwenk, Eur. Phys. J. A 50, 11 (2014).

[71] K. Tsukiyama, S. K. Bogner, and A. Schwenk, Phys. Rev. C 85, 061304(R) (2012).

[72] S. K. Bogner, H. Hergert, J. D. Holt, A. Schwenk, S. Binder, A. Calci, J. Langhammer, and R. Roth, arXiv:1402.1407

[73] G. R. Jansen, J. Engel, G. Hagen, P. Navrátil, and A. Signoracci, arXiv:1402.2563. 